\title{
Variable-density flow in porous media
}

\author{
By M. DENTZ $Z^{1}$, D. M. TARTAKOVSK $Y^{2,3}$, E. ABARCA ${ }^{1}$, \\ A. GUADAGNINI ${ }^{4}$, X. SANCHEZ-VILA AND J. CARRERA ${ }^{1}$ \\ ${ }^{1}$ Department of Geotechnical Engineering and Geosciences, Technical University \\ of Catalonia (UPC), Barcelona, Spain \\ ${ }^{2}$ Department of Mechanical \& Aerospace Engineering, University of California, \\ San Diego, La Jolla, USA \\ ${ }^{3}$ Theoretical Division, Los Alamos National Laboratory, Los Alamos, USA \\ ${ }^{4}$ DIIAR, Politecnico di Milano, Piazza L. da Vinci, 32, 20133, Milano, Italy
}

(Received 22 February 2005 and in revised form 11 January 2006)

Steady-state distributions of water potential and salt concentration in coastal aquifers are typically modelled by the Henry problem, which consists of a fully coupled system of flow and transport equations. Coupling arises from the dependence of water density on salt concentration. The physical behaviour of the system is fully described by two dimensionless groups: (i) the coupling parameter $\alpha$, which encapsulates the relative importance of buoyancy and viscous forces, and (ii) the Péclet number $P e$, which quantifies the relative importance of purely convective and dispersive transport mechanisms. We provide a systematic analytical analysis of the Henry problem for a full range of the Péclet number. For moderate $P e$, analytical solutions are obtained through perturbation expansions in $\alpha$. This allows us to elucidate the onset of density-driven vertical flux components and the dependence of the local hydraulic head gradients on the coupling parameter. The perturbation solution identifies the regions where salt concentration is most pronounced and relates their spatial extent to the development of a convection cell. Next, we compare our solution to a solution of the pseudo-coupled model, wherein flow and transport are coupled only via the boundary conditions. This enables us to isolate the effects caused by density-dependent processes from those induced by external forcings (boundary conditions). For small $P e$, we develop a perturbation expansion around the exact solution corresponding to $P e=0$, which sheds new light on the interpretation of processes observed in diffusion experiments with variable-density flows in porous media. The limiting case of infinite Péclet numbers is solved exactly for the pseudo-coupled model and compared to numerical simulations of the fully coupled problem for large $P e$. The proposed perturbation approach is applicable to a wide range of variable-density flows in porous media, including seawater intrusion into coastal aquifers and temperature or pressure-driven density flows in deep aquifers.

\section{Introduction}

Variable-density flow and transport in porous media have received increasing attention in the literature as fluid density variations play an important role in many environmental problems. One of the most significant environmental problems is that of pollution of freshwater bodies by water with high concentrations of salts in dissolution. Salty water can come either from the sea (e.g. Custodio, Bruggeman \& Cotecchia 1987) or from naturally occurring brines (e.g. Schelkes, Vogel \& Klinge 2001; Herbert, 
Jackson \& Lever 1988). High-density water can also result from landfill leachate and irrigation practices (e.g. Simmons et al. 2002). Another area of interest falling under the purview of variable-density flows is the problem of pollution by non-aqueousphase liquids (e.g. Taylor et al. 2001). All these problems concern flow in the shallow subsurface. Other studies deal with density-driven flow in the deep subsurface, where density differences are caused mainly by temperature or pressure. These variations can be both natural and anthropogenic. One example of the latter arises from nuclear fuel waste heat generation, which renders the study of variable-density flow relevant to performance assessment analyses (e.g. Ophori 2004; Yang \& Edwards 2000).

It is well known that variable-density flows in porous media can become unstable. Instabilities and fingering develop when a denser fluid lies above a lighter fluid (e.g. Manickam \& Homsy 1995; Wooding, Tyler \& White 1997). When lighter fluid is on top, flow is stable and mixing is caused by diffusive and/or dispersive mechanisms (e.g. Huppert \& Woods 1995). This is the flow scenario that usually occurs during seawater intrusion into coastal aquifers (e.g. Alkalali \& Rostron 2003) and is the subject of this study. This problem poses significant environmental and economical challenges around the world (Panday et al. 1993; Smith \& Turner 2001; Aliewi et al. 2001; Paniconi et al. 2001) and has important water resources management implications because relatively a small proportion of seawater (about $1 \%$ ) will render freshwater unfit for drinking.

Seawater intrusion has been traditionally described by two alternative mathematical

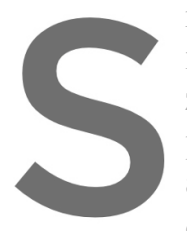
models. The first fresh water from zone, this approach propagation. The analytical solution \& Ouazar 1998

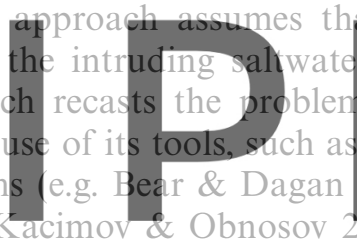
that a sharp in
ar. By ignorin
s conformal m
1964; Huppe
2001), which prems of DI A the phenomenon. There exist, however, a plethora of physical conditions for which

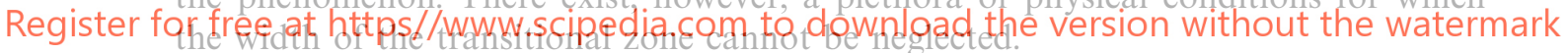

The second approach, which we adopt here, accounts for the presence of the transition zone resulting from the dynamic equilibrium of moving fresh- and saltwater. The corresponding mathematical model consists of a system of the variable-density (Darcy) flow equation and the advection-dispersion/diffusion equation. The two governing equations are fully coupled through the dependence of water density on salt concentration.

The intrinsic complexity of the coupled governing equations precludes the development of closed-form analytical solutions even for simple geometries and boundary conditions. This, in turn, has led to the proliferation of numerical codes, many of which are reviewed in Bear et al. (1999). The absence of analytical solutions and the relative scarcity of experimental data (Schincariol \& Schwartz 1990; Oltean et al. 2004) complicates the establishment of benchmark problems for testing the accuracy and robustness of these numerical codes. Instead, numerical solutions are compared to each other, and the differences between them can be quite significant (Croucher \& O'Sullivan 1995). Therefore, there is a need to provide analytical solutions for specific problems that can be used for benchmarking (Weatherill et al. 2004).

This study is devoted to the analysis of the Henry problem (Henry 1964), which, despite some reservations (Simpson \& Clement 2003), remains one of the most widely used benchmark problems. The importance of the Henry problem goes beyond benchmarking, since it provides physical insight into transport processes associated with seawater intrusion (Abarca et al. 2005). Available quasi-analytical solutions of 
the Henry problem (Henry 1964; Segol 1994) are based on a Galerkin method and are rendered impractical by their slow rates of convergence. Typically, the Henry problem is solved numerically (Pinder \& Cooper 1970; Segol, Pinder \& Gray 1975; Galeati, Gambolati \& Neuman 1992; Segol 1994), leading to quantitatively different solutions (Croucher \& O’Sullivan 1995).

In this study we present a new methodology to solve analytically the Henry problem and other similar problems describing variable-density flows in porous media. Section 2 contains a mathematical formulation of the Henry problem and introduces relevant dimensionless parameters. In $\S 3$, we provide an exact solution for the flow problem as a functional of the salt concentration, which serves as a basis for a perturbation solution of the fully coupled system of variable-density flow equations for weak density coupling $(\S 4)$ and small Péclet numbers $(\S 5)$. Section 6 studies flow and transport in the opposite limit of large Péclet numbers. The limit of infinite Péclet numbers, i.e. zero diffusion, corresponds to the case of a sharp interface between fresh- and saltwater.

\section{The Henry problem: steady-state flow and transport}

\subsection{Problem formulation}

The Henry formulation of seawater intrusion describes the steady-state position of a diffused saltwater wedge within a confined aquifer balanced against a flowing freshwater field. Within this framework, fluid flow in porous media is governed by

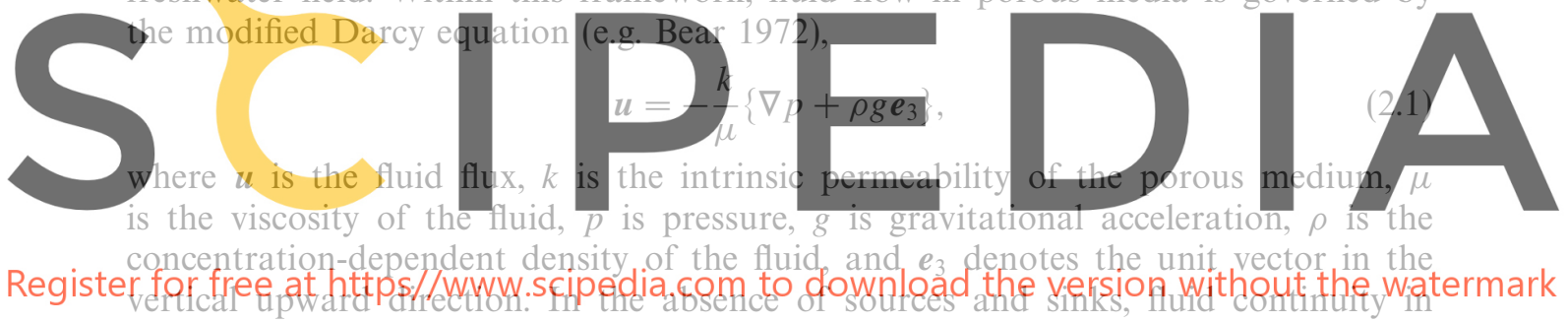
steady state is expressed by

$$
\nabla \cdot\{\rho \boldsymbol{u}\}=0 .
$$

The fluid density $\rho$ varies with the salt concentration $c$, whose dynamics satisfy the steady-state advection-diffusion equation (Henry 1964)

$$
\nabla \cdot\{\boldsymbol{u} c-\theta D \nabla c\}=0,
$$

where $D$ is the constant diffusion coefficient. For homogeneous porous media, both permeability $k$ and porosity $\theta$ are constant.

Equations (2.1) $-(2.3)$ are closed by specifying a constitutive relationship $\rho=\rho(c)$ between the fluid density $\rho$ and the salt concentration $c$. While a number of such relationships exists (e.g. Holzbecher 1998; Diersch \& Kolditz 2002), the linear relationship

$$
\rho=\rho_{f}\left(1+\epsilon \frac{c}{c_{s}}\right)
$$

originally used in Henry (1964) remains the most popular. Here $c_{s}$ is the concentration of salt in seawater and

$$
\epsilon \equiv \frac{\rho_{s}-\rho_{f}}{\rho_{f}}
$$

denotes the relative density contrast between the densities of freshwater $\left(\rho_{f}\right)$ and saltwater $\left(\rho_{s}\right)$. Note that $c \leqslant c_{s}$ and, hence, $\rho_{f} \leqslant \rho \leqslant \rho_{s}$. 


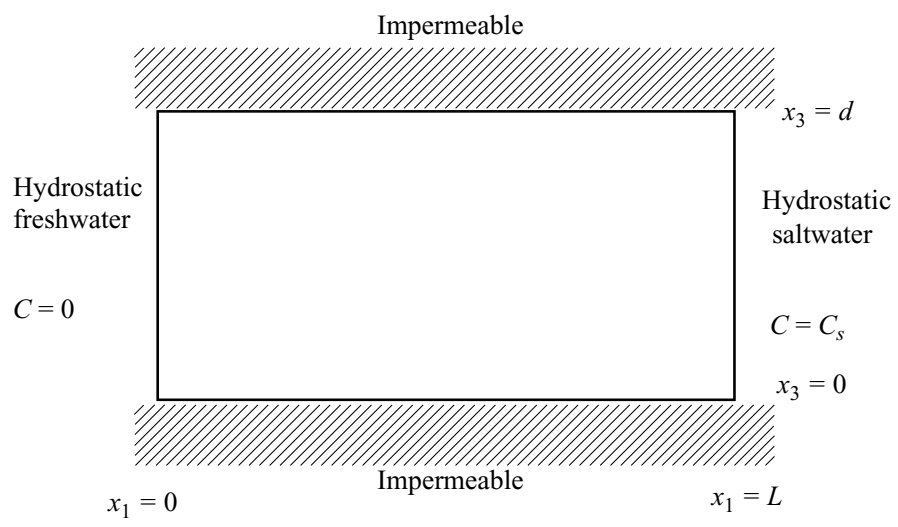

FIGURE 1. Illustration of the flow and transport scenario (after Croucher \& O'Sullivan 1995). Pressure is hydrostatic at the inland $\left(x_{1}=0\right)$ and sea $\left(x_{1}=L\right)$ boundaries. The salt concentration is zero at the freshwater boundary, and equal to the (constant) concentration $C_{s}$ of salt in seawater at the sea boundary. The top and bottom of the domain are impermeable.

For the usual range of temperatures in aquifers, the density of freshwater is $\rho_{f}=10^{3} \mathrm{~kg} \mathrm{~m}^{-3}$. The density of seawater generally increases with decreasing temperature, increasing salinity, and increasing pressure. With a few exception the density of seawater and $1.029 \mathrm{~kg} \mathrm{~m}^{-3}$, typical ocean conditions, Diersch \& Kolditz 2002)

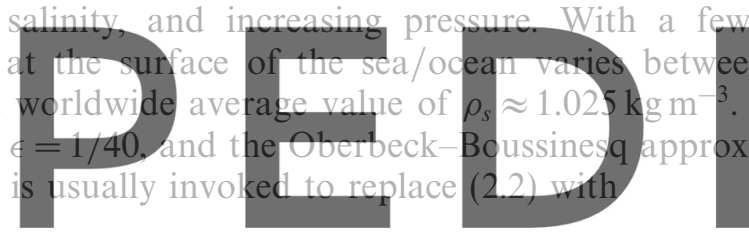

$\nabla \cdot u=0$.

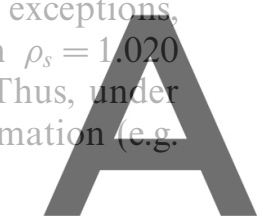

(2.6)

Register for free at https//www.scipedia.com to download the version without the watermark A justification is that the average direction of flow in steady aquifers is nearly orthogonal to that of the density gradient, i.e. $\boldsymbol{u} \cdot \nabla \rho \approx 0$.

The Henry problem (Henry 1964) and its subsequent modifications (Segol 1994; Croucher \& O'Sullivan 1995) deal with the two-dimensional versions of equations (2.1), (2.3), (2.4), and (2.6), which are defined on a vertical cross-section of an aquifer, i.e. $\boldsymbol{x} \equiv\left(x_{1}, x_{3}\right)^{T} \in \Omega$, where $\Omega=[0, L] \times[0, d]$ is a rectangle shown in figure 1 . In this formulation, the horizontal boundaries $x_{3}=0$ and $x_{3}=d$ are assumed to be impermeable to both flow and transport. Freshwater enters the aquifer $\Omega$ through the inland boundary $x_{1}=0$ and discharges into the ocean through the coastal boundary $x_{1}=L$. Saltwater enters the aquifer $\Omega$ through the coastal boundary $x_{1}=L$, advances, and mixes with the discharging freshwater. This causes the formation of a diffused transitional zone that separates freshwater from seawater.

An immediate consequence of this formulation is that both freshwater and mixed water discharge into the ocean through the coastal boundary $x_{1}=L$. This is in contrast to sharp-interface formulations, where the presence of the interface between freshand saltwater gives rise to a vortex-like singularity on the $x_{1}=L$ boundary. This issue is discussed further in $\S 6$.

The corresponding boundary conditions for flow equations (2.1) and (2.6) are as follows. Fluid pressure $p(\boldsymbol{x})$ is prescribed at the freshwater and saltwater boundaries, where it is given by the pressure of hydrostatic saltwater. This yields

$$
p\left(x_{1}=0, x_{3}\right)=p_{0}-\rho_{f} g x_{3}, \quad p\left(x_{1}=L, x_{3}\right)=\rho_{s} g\left(d-x_{3}\right)
$$


where $p_{0}$ is the pressure at the bottom of the inland boundary. At the impermeable top and bottom, the vertical component of the Darcian velocity $\boldsymbol{u}=\left(u_{1}, u_{3}\right)^{T}$ is zero,

$$
u_{3}\left(x_{1}, x_{3}=0\right)=0, \quad u_{3}\left(x_{1}, x_{3}=d\right)=0 .
$$

The boundary conditions for transport equation (2.3) are as follows. At the freshwater and sea boundaries the salt concentration is

$$
c\left(x_{1}=0, x_{3}\right)=0, \quad c\left(x_{1}=L, x_{3}\right)=c_{s},
$$

respectively. At the impermeable horizontal boundaries, mass flux is zero,

$$
\frac{\partial c\left(x_{1}, x_{3}=0\right)}{\partial x_{3}}=0, \quad \frac{\partial c\left(x_{1}, x_{3}=d\right)}{\partial x_{3}}=0 .
$$

Note that the existence of a steady-state regime described by the Henry model requires both that the diffused freshwater-saltwater interface be in dynamical equilibrium with average sea level, and that temporal variations in the total flux of freshwater be negligible. This steady-state situation of dynamical equilibrium does occur in many coastal aquifers (e.g. Bear 1972; Bear et al. 1999).

The original Henry problem (Henry 1964) was formulated in terms of stream functions. Henry implicitly imposed the inland boundary to represent hydrostatic

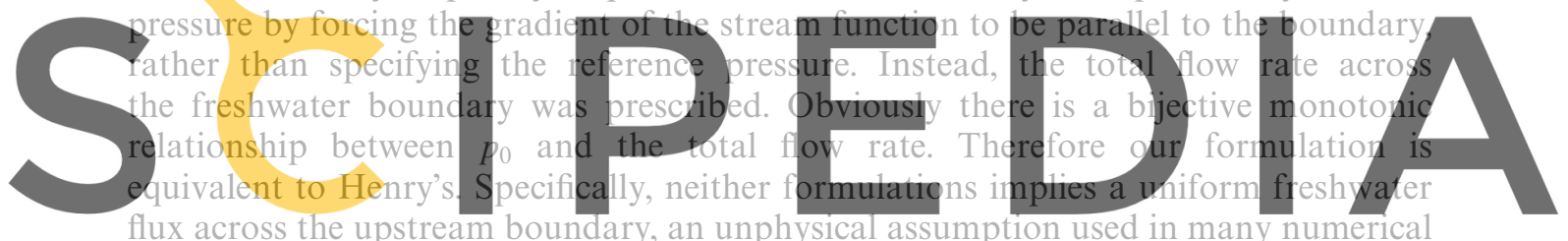

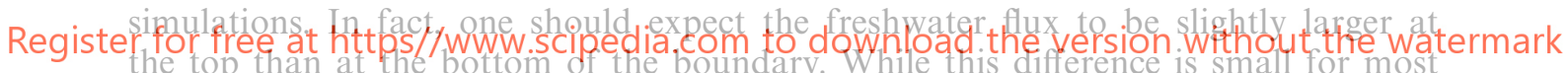
problems, it must be kept in mind when conducting detailed comparisons.

\subsection{Freshwater head formulation}

We start our analysis by reformulating the generalized Darcy law (2.1) in terms of the equivalent freshwater hydraulic head

$$
h=\frac{p}{\rho_{f} g}+x_{3} .
$$

Substituting (2.4) and (2.9) into (2.1) gives

$$
\boldsymbol{u}=-K\left(\nabla h+\boldsymbol{e}_{3} \epsilon \frac{c}{c_{s}}\right), \quad K \equiv \frac{k \rho_{f} g}{\mu},
$$

where $K$ is the hydraulic conductivity of a porous medium. Combining (2.6) and (2.10) yields the flow equation written in terms of the freshwater head $h$,

$$
\nabla^{2} h=-\frac{\epsilon}{c_{s}} \frac{\partial c}{\partial x_{3}} .
$$

In terms of hydraulic head (2.9), boundary conditions (2.7a) and (2.7b) are recast as 


$$
h\left(x_{1}=0, x_{3}\right)=\frac{p_{0}}{\rho_{f} g} \equiv h_{0}, \quad h\left(x_{1}=L, x_{3}\right)=d+\epsilon\left(d-x_{3}\right),
$$

and

$$
\frac{\partial h\left(x_{1}, x_{3}=0\right)}{\partial x_{3}}=-\epsilon \frac{c\left(x_{1}, x_{3}=0\right)}{c_{s}}, \quad \frac{\partial h\left(x_{1}, x_{3}=d\right)}{\partial x_{3}}=-\epsilon \frac{c\left(x_{1}, x_{3}=d\right)}{c_{s}},
$$

respectively.

Substituting (2.6) and (2.10) into (2.3) leads to the steady-state transport equation expressed in terms of the freshwater head $h$,

$$
K \nabla h \cdot \nabla c+K \frac{c}{c_{s}} \frac{\partial c}{\partial x_{3}}+\theta D \nabla^{2} c=0
$$

Equation (2.13) is subject to the boundary conditions (2.8).

\subsection{Dimensionless form of the governing equations}

Next, we recast the governing equations (2.11) and (2.13) in a dimensionless form. Let $\xi=\left(\xi_{1}, \xi_{3}\right)^{T}$ and $\zeta$ denote the rescaled (dimensionless) spatial position vector the dimensionless aquifer thickness,

$$
\xi_{1}=\frac{x_{1}}{L}, \quad \xi_{3}=\frac{x_{3}}{L}, \quad \zeta=\frac{d}{L},
$$

respectively. This transformation maps the flow domain $\Omega=[0, L] \times[0, d]$ onto $\Omega_{d}=[0,1] \times[0, \zeta$. From now $\mathrm{Qn}, \nabla$ is understood to operate with respect to
the dimensionless coordinates $\xi$. Dimensionless freshwater hydraulid head $H$, salt
concentration $C$ and Darcy velocity $U$ are derined by
\[ H(\xi)=\frac{h(L \xi)-d}{\Delta h}, \quad C(\xi)=\frac{c(L \xi)}{c}, \quad \boldsymbol{U}(\xi)=\frac{u(L \xi) L}{K \Delta h_{0}}, \]

where

Register for free at https//www.scipedia.com to download the version without the watermark

is the global head difference imposed by the inland and sea boundaries in the absence of density effects. Finally, we introduce dimensionless parameters

$$
\alpha=\frac{\epsilon L}{\Delta h_{0}}, \quad P e=\frac{K \Delta h_{0}}{\theta D} .
$$

With these definitions the dimensionless Darcy velocity $\boldsymbol{U}(\xi)=\left(U_{1}, U_{3}\right)^{T}$ is given by

$$
\boldsymbol{U}(\xi)=-\left\{\nabla H(\xi)+\boldsymbol{e}_{3} \alpha C(\xi)\right\} .
$$

This leads to the dimensionless formulation of the salt transport equation (2.3),

$$
\boldsymbol{U} \cdot \nabla C-P e^{-1} \nabla^{2} C=0 .
$$

The flow problem (2.11) and (2.12) becomes

$$
\nabla^{2} H(\xi)=-\alpha \frac{\partial C(\xi)}{\partial \xi_{3}}
$$

subject to the boundary conditions

$$
\begin{gathered}
H\left(0, \xi_{3}\right)=1, \quad H\left(1, \xi_{3}\right)=\alpha\left(\zeta-\xi_{3}\right), \\
\frac{\partial H\left(\xi_{1}, 0\right)}{\partial \xi_{3}}=-\alpha C\left(\xi_{1}, 0\right), \quad \frac{\partial H\left(\xi_{1}, \zeta\right)}{\partial \xi_{3}}=-\alpha C\left(\xi_{1}, \zeta\right) .
\end{gathered}
$$


Combining (2.18) and (2.19) recasts the transport problem (2.13) and (2.8) as

$$
\nabla H(\xi) \cdot \nabla C(\xi)+\alpha C(\xi) \frac{\partial C(\xi)}{\partial \xi_{3}}+P e^{-1} \nabla^{2} C(\xi)=0
$$

subject to the boundary conditions

$$
\begin{array}{rlrl}
C\left(0, \xi_{3}\right) & =0, & & C\left(1, \xi_{3}\right)=1, \\
\frac{\partial C\left(\xi_{1}, \xi_{3}=0\right)}{\partial \xi_{3}}=0, & & \frac{\partial C\left(\xi_{1}, \xi_{3}=\zeta\right)}{\partial \xi_{3}}=0 .
\end{array}
$$

It now becomes apparent that the Henry problem for seawater intrusion in coastal aquifers (2.20)-(2.23) is completely characterized by the dimensionless parameters $\alpha$ and $P e$ in (2.17). The coupling parameter $\alpha$ is the ratio of the characteristic velocity induced by density effects, $K \epsilon$, to the flow velocity in the absence of density effects, $K \Delta h_{0} / L$, and can also be viewed as the ratio of buoyancy to viscous forces. The strength of coupling between the flow and salt transport processes increases with $\alpha$. The Péclet number $P e$ is the ratio between a typical water flux in the absence of density effects, $K \triangle h_{0}$ to diffusion in the porous medium, $\theta D$, and as such compares the relative importance of advective and diffusive transport mechanisms.

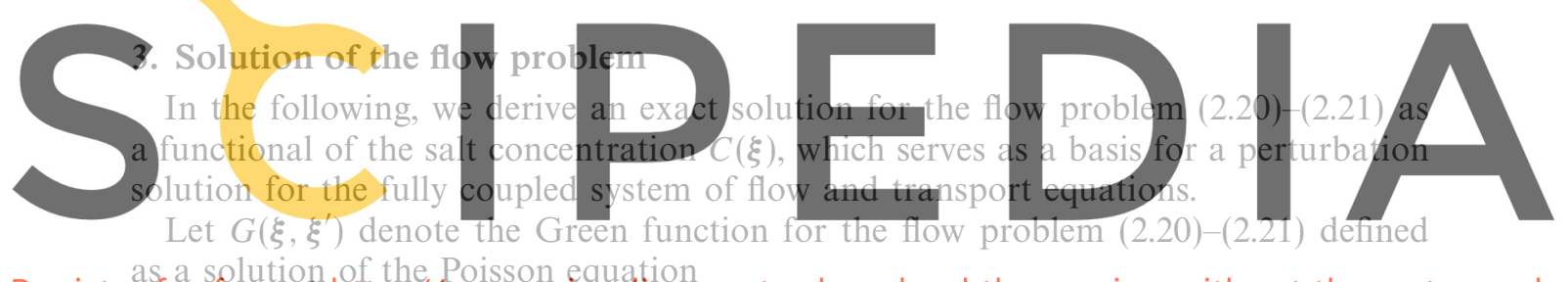
Register for free at hittps//Www.scipedia.com to download the version without the watermark

$$
\nabla^{2} G\left(\xi, \xi^{\prime}\right)=-\delta\left(\xi-\xi^{\prime}\right),
$$

where $\delta(\xi)$ is the Dirac delta function, subject to the homogeneous boundary conditions

$$
G_{\left.\right|_{\xi_{1}=0}=G_{\mid \xi_{1}=1}}=0, \quad \frac{\partial G}{\partial \xi_{3}}=\frac{\partial G}{\partial \xi_{\xi_{3}=0}}=0 .
$$

Then a formal solution of (2.20)-(2.21) can be written as

$$
H(\boldsymbol{\xi})=\alpha \int_{\Omega_{d}} G \frac{\partial c}{\partial \xi_{3}^{\prime}} \mathrm{d} \boldsymbol{\xi}^{\prime}+\int_{\partial \Omega_{d}}\left[\boldsymbol{e}_{3} G \frac{\partial H}{\partial \xi_{3}^{\prime}}-\boldsymbol{e}_{1} H \frac{\partial G}{\partial \xi_{1}^{\prime}}\right] \cdot \mathrm{d} \boldsymbol{n},
$$

where $\boldsymbol{n}$ is the unit normal vector for the domain boundary $\partial \Omega_{d}$.

The expressions for $G\left(\xi, \xi^{\prime}\right)$ in $(3.1 a)-(3.1 b)$ are given in terms of double infinite summations (e.g. Carslaw \& Jaeger 1959; Butkovskii 1982). In the Appendix § A.1, we derive two alternative representations for the Green function $G\left(\xi, \xi^{\prime}\right)$, both of which involve single infinite summations. We use the first representation (A 7) to evaluate the first boundary integral on the right-hand side of (3.2) and the second representation (A 10) to evaluate the second boundary integral. Thus, (3.2) gives a solution for the dimensionless freshwater head,

$$
H(\xi)=\left(1-\xi_{1}\right)+\alpha \eta_{B}(\xi)+\alpha \eta_{C}[\xi, C(\xi)],
$$


where

$$
\eta_{B}=\frac{\xi_{1} \zeta}{2}+\sum_{l=1}^{\infty} a_{l} \cos \left(\frac{l \pi \xi_{3}}{\zeta}\right) \frac{\sinh \left(l \pi \xi_{1} / \zeta\right)}{\sinh (l \pi / \zeta)}, \quad a_{l}=\frac{2 \zeta\left(1-(-1)^{l}\right)}{l^{2} \pi^{2}},
$$

represents a contribution to $H$ stemming from the boundary conditions at the saltwater boundary, and

$$
\begin{gathered}
\eta_{C}=\int_{\Omega_{d}} G \frac{\partial C}{\partial \xi_{3}^{\prime}} \mathrm{d} \xi^{\prime}+\sum_{l=1}^{\infty} \frac{1}{l \pi} \frac{\sin \left(l \pi \xi_{1}\right)}{\sinh (l \pi \zeta)}\left\{\tilde{b}_{l} \cosh \left[l \pi\left(\xi_{3}-\zeta\right)\right]-\hat{b}_{l} \cosh \left(l \pi \xi_{3}\right)\right\}, \\
\tilde{b}_{l}=2 \int_{0}^{1} C\left(\xi_{1}, 0\right) \sin \left(l \pi \xi_{1}\right) \mathrm{d} \xi_{1}, \quad \hat{b}_{l}=2 \int_{0}^{1} C\left(\xi_{1}, \zeta\right) \sin \left(l \pi \xi_{1}\right) \mathrm{d} \xi_{1},
\end{gathered}
$$

is a contribution to $H$ resulting entirely from the coupling of flow and salt transport.

The solution (3.3) is formal, in that it depends on the yet unknown salt concentration distribution $C(\xi)$. In $\$ 4$, we use a perturbation expansion in the coupling parameter $\alpha$ to oyercome this problem. An alternative to this approach is to use simplifying physical assumptions that give rise to the pseudo-coupled transport model described below.

\subsection{Pseudo-coupled transport model}
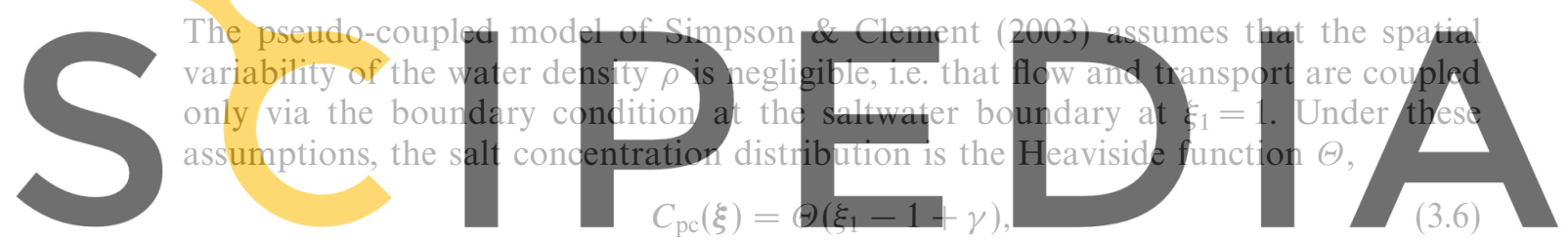

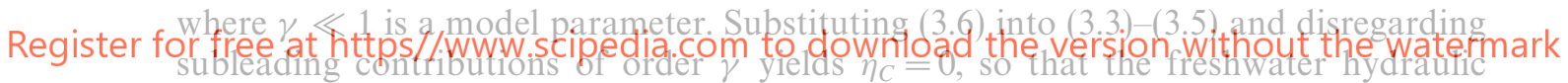

head for the pseudo-coupled model is given by

$$
H_{\mathrm{pc}}(\xi)=1-\xi_{1}+\alpha \eta_{B}(\xi) \text {. }
$$

One of the goals of our analysis is to examine the validity and accuracy of the pseudo-coupled model for variable-density flow in porous media.

\section{Intermediate Péclet numbers}

Here we use perturbation expansions in the coupling parameter $\alpha$ to derive approximate analytical solutions for the freshwater hydraulic head and the salt concentration. Since this requires $\alpha$ to be small, it is worth revisiting the physical implications of this requirement.

Since the coupling parameter $\alpha$ compares a typical flow velocity induced by density contrasts at the saltwater boundary to the flow velocity without density effects, it affect variable-density flow in porous media in two important ways. First, in the absence of density contrasts, flow, induced by hydrostatic saltwater at the boundary $\xi_{1}=1$, is horizontal. Hence, $\alpha$ serves as a measure of the deviation of the flow velocity from the $\xi_{1}$-direction. The magnitude of this deviation is quantified by $\eta_{B}(\xi)$ in (3.4). Second, $\alpha$ quantifies the influence of density effects on the flow velocity due to intruding saltwater. As such, it is a measure of the strength of the coupling between fluid flow and salt transport, as quantified by $\eta_{C}[\xi, C(\xi)]$ in (3.5). 
We begin our perturbation analysis by recasting the transport problem (2.22) in the form of an integral equation.

\subsection{Integral equation for the transport problem}

Substituting (3.3) into (2.22) leads to the nonlinear transport equation

$$
\frac{\partial C}{\partial \xi_{1}}-P e^{-1} \nabla^{2} C=\alpha \nabla \eta_{B} \cdot \nabla C+\alpha\left(\nabla \eta_{C} \cdot \nabla+C \frac{\partial}{\partial \xi_{3}}\right) C .
$$

Let $g\left(\xi, \xi^{\prime}\right)$ be the Green function defined as the solution of the advection-diffusion equation

$$
P e^{-1} \nabla^{2} g\left(\xi, \xi^{\prime}\right)-\frac{\partial g\left(\xi, \xi^{\prime}\right)}{\partial \xi_{1}}=-\delta\left(\xi-\xi^{\prime}\right),
$$

subject to homogeneous boundary conditions

$$
g_{\xi_{1}=0}=g_{\xi_{1}=1}=0, \quad \frac{\partial g}{\partial \xi_{3}}=\frac{\partial g}{\partial \xi_{\xi_{3}=0}}=0 .
$$

The corresponding analytical expression for $g\left(\xi, \xi^{\prime}\right)$ in the form of a single infinite summation is derived in the Appendix $\S$ A.2.

Rewriting (4.1) in terms of $\xi^{\prime}$, multiplying the result with $g\left(\xi, \xi^{\prime}\right)$, integrating over $\Omega_{d}$, and applying the Green theorem yields a nonlinear integral equation

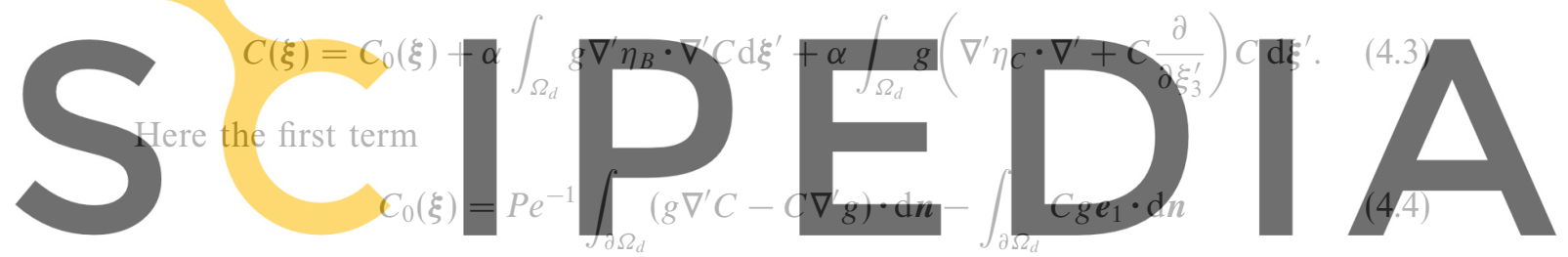

Register for free for the boundary effects on the salt concentration distribution Ch the the second term rellects the influence of the hydrostatic saltwater boundary on salt transport, and the third term quantifies the linear coupling between flow and transport.

It is interesting to note that (4.4) is the solution of the transport problem (4.1) and (2.23) in the absence of density effects, i.e. when $\alpha=0$. Substituting (A 24) into (4.4), while recalling boundary conditions $(2.23)$, gives

$$
C_{0}(\xi) \equiv C_{0}\left(\xi_{1}\right)=\frac{\exp \left(\xi_{1} P e\right)-1}{\exp (P e)-1} .
$$

We solve the system of nonlinear equations (3.3) and (4.3) analytically via a perturbation expansion in the coupling parameter $\alpha$.

\subsection{Perturbation solution}

Consider an expansion of the salt concentration $C(\xi)$ into a perturbation series in powers of $\alpha$,

$$
C(\xi)=\sum_{k=0}^{\infty} \alpha^{k} C^{(k)}(\xi)
$$

which implies that the coupling parameter $\alpha$ is small. Substituting (4.6) into (3.5) gives

$$
\eta_{C}\left[\boldsymbol{\xi}, \sum_{k=0}^{\infty} \alpha^{k} C^{(k)}(\boldsymbol{\xi})\right]=\sum_{k=0}^{\infty} \alpha^{k} \eta_{C}\left[\boldsymbol{\xi}, C^{(k)}(\boldsymbol{\xi})\right]
$$


since $\eta_{C}[\xi, C(\xi)]$ is linear in $C(\xi)$. Then it follows from (3.3) that hydraulic head $H$ can be represented by a perturbation series

$$
H(\xi)=\sum_{k=0}^{\infty} \alpha^{k} H^{(k)}(\xi)
$$

Substituting (4.7) into (3.3) gives an explicit expression for an expansion of $H(\xi)$ in terms of the expansion of $C(\xi)$ in (4.6),

$$
H(\xi)=1-\xi_{1}+\alpha\left\{\eta_{B}(\xi)+\eta_{C}\left[\xi, C^{(0)}(\xi)\right]\right\}+\sum_{k=2}^{\infty} \alpha^{k} \eta_{C}\left[\xi, C^{(k-1)}(\xi)\right] .
$$

Comparison of (4.8) and (4.9) shows that

$$
\left.\begin{array}{ll}
H^{(0)}(\xi)=1-\xi_{1}, & H^{(1)}(\xi)=\eta_{B}(\xi)+\eta_{C}\left[\xi, C^{(0)}(\xi)\right], \\
H^{(k)}(\xi)=\eta_{C}\left[\xi, C^{(k-1)}(\xi)\right], \quad k>1 .
\end{array}\right\}
$$

Equations (4.10) reveal that the terms $H^{(k)}$ with $k>1$ in the expansion (4.8) depend on the terms $C^{(k-1)}$ in the expansion (4.6).

Substituting (4.6) and (4.7) into (4.3) we obtain
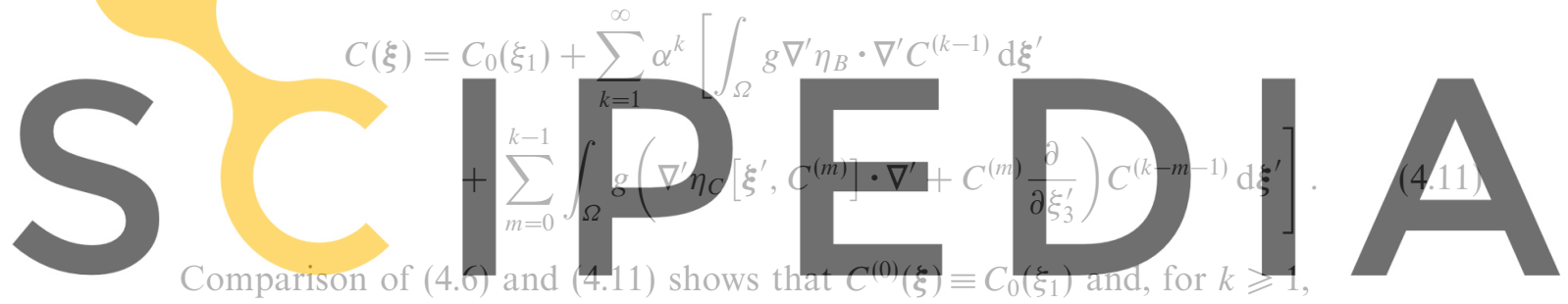

Register for free at dittps/_Lwyw. scipedia.com do download the version without the watermark

$$
+\sum_{m=0}^{k-1} \int_{\Omega} g\left(\nabla^{\prime} \eta_{C}\left[\xi^{\prime}, C^{(m)}\right] \cdot \nabla^{\prime}+C^{(m)}\left(\xi^{\prime}\right) \frac{\partial}{\partial \xi_{3}^{\prime}}\right) C^{(k-m-1)} \mathrm{d} \xi^{\prime}
$$

Thus our perturbation expansions generate a hierarchy of recursive relations for $C^{(k)}(\xi)$, which in turn determine the terms $H^{(k+1)}(\xi)$ in the expansion of the hydraulic head $H(\xi)$. Thus, the fully coupled system of equations for flow and transport has been decoupled by means of an expansion in the coupling parameter $\alpha$.

Nonlinearity of the transport equation (4.1), caused by the dependence of $H$ on the salt concentration, manifests itself through dependence of the expansion terms $C^{(k)}(\xi)$ with $k \geqslant 1$ on all $C^{(j)}(\xi)$ with $j \in[1, \ldots, k-1]$. Hence, every approximation order in $\alpha$ simultaneously increases the accuracy of the solutions for $C$ as well as for $H$.

\subsubsection{Perturbation solution of the pseudo-coupled model}

For the pseudo-coupled model (3.6) and (3.7), the transport equation (4.1) is linear in $C(\xi)$, the second term on the right-hand side of (4.12) is zero, and each $C^{(k)}(\xi)$ with $k \geqslant 1$ in (4.12) depends only on the lower-order expansion term $C^{(k-1)}(\xi)$. Hydraulic head $H(\xi)$ is exact at first order in $\alpha$. In this case, the perturbation solution represents an expansion in the deviations of the exactly known flow field from constant uniform flow in the $\xi_{1}$-direction, and every approximation order increases the accuracy of the concentration solution. 


\subsection{First-order approximations and numerical simulations}

In the following, we evaluate expressions (4.6) and (4.8) up to first order in $\alpha$. We consider both the coupled and pseudo-coupled formulations of the flow and transport problems and compare their solutions with numerical simulations obtained from the well-tested and widely used software SUTRA (Voss \& Provost 2002).

The first-order approximations of hydraulic head $H(\xi)$ and salt concentration $C(\xi)$ are given by

$$
H(\xi)=1-\xi_{1}+\alpha \eta_{B}(\xi)+\alpha \eta_{C}\left[\xi, C_{0}(\xi)\right]+O\left(\alpha^{2}\right)
$$

and

$C(\xi)=C_{0}\left(\xi_{1}\right)+\alpha \int_{\Omega_{d}} g \frac{\partial \eta_{B}}{\partial \xi_{1}^{\prime}} \frac{\partial C_{0}}{\partial \xi_{1}^{\prime}} \mathrm{d} \xi^{\prime}+\alpha \int_{\Omega_{d}} g \frac{\partial \eta_{C}\left[\xi^{\prime}, C_{0}\left(\xi_{1}^{\prime}\right)\right]}{\partial \xi_{1}^{\prime}} \frac{\partial C_{0}}{\partial \xi_{1}^{\prime}} \mathrm{d} \xi^{\prime}+O\left(\alpha^{2}\right)$,

respectively. The coefficients $\tilde{b}_{l}$ and $\hat{b}_{l}$ in the expression for $\eta_{C}$ in (3.5) are evaluated explicitly to yield

$$
\tilde{b}_{l} \equiv \hat{b}_{l}=\frac{2}{\exp (P e)-1}\left\{l \pi \frac{1-\exp (P e)(-1)^{l}}{P e^{2}+(l \pi)^{2}}+\frac{(-1)^{l}-1}{l \pi}\right\} .
$$

The infinite summations in the expressions for the Green function $g$ in (A 24), $\eta_{B}$ in (3.4) and $\eta_{C}$ in (3.5) converge exponentially with the summation index. Their numerical evaluation requires a relatively small number of terms $(10$, in our numerical calculations). The quadratures in (4.14) and (4.16) are evaluated numerically with a ten-point Gauss-Legendre integration (Press et al. 1992).

\subsubsection{First-order solutions of the pseudo-coupled problem}

For the pseudo-coupled problem, the first-order expression for hydraulic head $H_{\mathrm{pc}}(\xi)$ is given by (3.7). The first-order approximation of the salt concentration distribution is given by

$$
C_{\mathrm{pc}}(\xi)=C_{0}\left(\xi_{1}\right)+\alpha \int_{\Omega_{d}} g \frac{\partial \eta_{B}}{\partial \xi_{1}^{\prime}} \frac{\partial C_{0}}{\partial \xi_{1}^{\prime}} \mathrm{d} \xi^{\prime}+O\left(\alpha^{2}\right)
$$

Comparison of (3.7) to (4.13) and (4.14) to (4.16) shows that the pseudo-coupled model results in the first-order solutions for hydraulic head $H$ and salt concentration $C$ that have the same leading behaviour as their counterparts derived from the coupled model. However, the sub-leading terms in these expressions are incomplete.

\subsubsection{Direct numerical simulations}

Direct numerical simulations of the flow and transport problems (2.20)-(2.23) were performed with SUTRA (Voss \& Provost 2002), a finite element code for solving a system of partial differential equations for variable-density flow and transport. The calculations for the decoupled and coupled transport problems were performed with a spatial resolution of $\Delta \xi_{1}=\Delta \xi_{3}=5 \times 10^{-3}$, which corresponds to $400 \times 200$ elements. For the pseudo-coupled problem, heads and concentrations were obtained by solving the steady-state flow and transport equations. For the coupled problem, heads and concentrations were determined iteratively as an asymptotic limit of the respective transient solutions. The solutions were asymptotically stationary and did not change after a simulation time of $6 \times 10^{5} \mathrm{~s}$.

In the following simulations, we set a thickness $\zeta=0.5$ and the Péclet number $P e=10$. The former corresponds to the original shape of the flow domain in the Henry problem. The latter represents a value for the Péclet number for which the intrusion of seawater is noticeable without being dominated by diffusion. We 
(a)

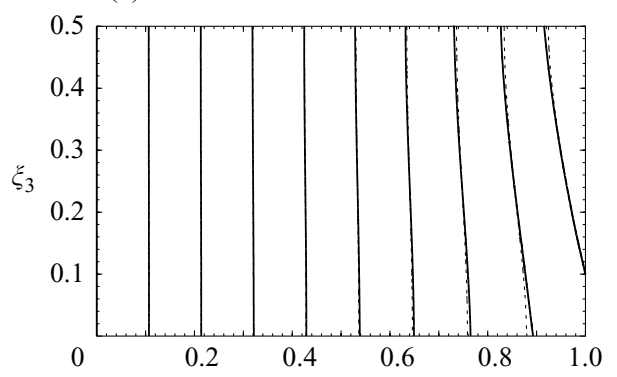

(c)

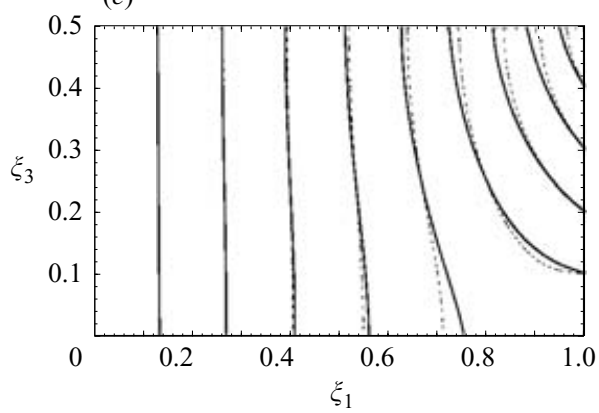

(b)

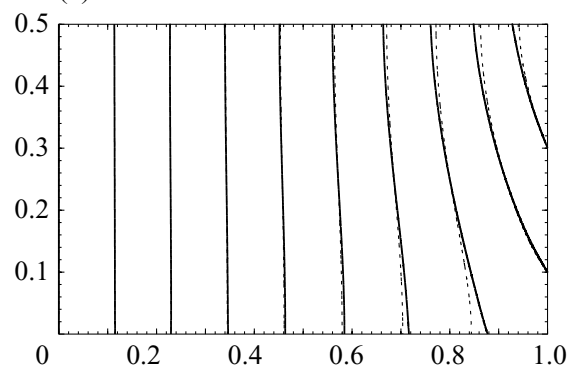

(d)

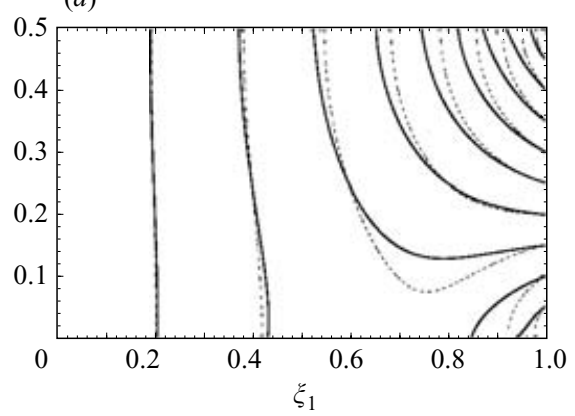

FIGURE 2. Equipotential lines of the first-order approximation of dimensionless freshwater head $H(\xi)$ corresponding to the coupled model (solid lines) given by (4.13) and to the pseudo-coupled model (dotted lines) given by (3.7), for $\zeta=0.5, P e=10$, and $(a) \alpha=0.25,(b) \alpha=$ $0.5,(c) \alpha=1.0$, and $(d) \alpha=2.0$. The isolines are equidistant with $\Delta H=0.1$.

considered a range of values of the coupling parameter $\alpha$ varying from $\alpha=0.25$ to 2.0. Since we set $\epsilon=1 / 40$, the lowest value corresponds to a freshwater hydraulic head gradient of $10 \%$, which should be considered as high, while the other extreme $(\alpha=2)$ corresponds to a freshwater hydraulic head gradient of $1.25 \%$, which is typical for many coastal aquifers.

\subsection{Freshwater head}

Figure 2 illustrates the impact of the modelling assumptions by comparing the first-order solutions (3.7) and (4.13) for the pseudo-coupled and coupled flow and transport models, respectively. For weak coupling $(\alpha=0.25$ and 0.5$)$, the solutions for the hydraulic head $H(\xi)$ resulting from the two models coincide at some distance from the saltwater boundary $\xi_{1}=1$, while differing visibly in the region adjacent to this boundary. Hydraulic head is dominated by the saltwater boundary conditions, yet visible density effects due to the intruding saltwater are apparent. The head isolines in the coupled model are more curved than their pseudo-coupled counterparts. An increasing vertical component of the flow velocity reflects the effect of buoyancy which causes light freshwater to tend to float on top of denser saltwater. Still, boundary heads dominate the solution, so that water flows from the inland freshwater boundary to the sea through the whole aquifer cross-section.

For stronger coupling ( $\alpha=1$ and 2), the differences between the head isolines for the two models become more pronounced. The distance between the isolines in the left half of the flow domain increases compared to the weaker coupling, which indicates a decreasing freshwater flow. The head isolines connecting the bottom of the flow domain with the saltwater boundary indicate a convection cell developing in the lower 

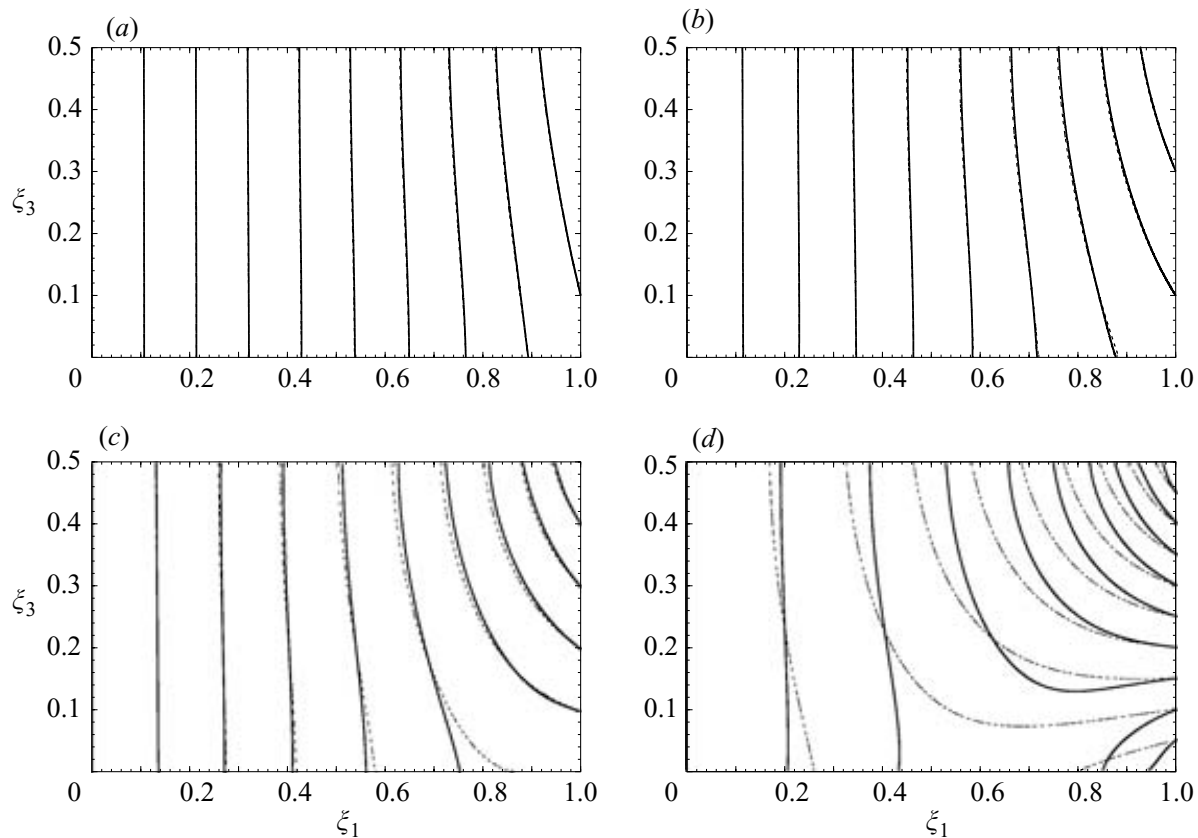

FIGURE 3. Equipotential lines of the first-order approximation of dimensionless freshwater head $H(\xi)$ resulting from the coupled model (solid lines) given by (4.13) and of the numerical solution of the full problem (2.20)-(2.23) obtained with SUTRA (dotted lines), for $\zeta=0.5$, $P e=10$, and $(a) \alpha=0.25,(b) \alpha=0.5,(c) \alpha=1.0$, and $(d) \alpha=2.0$. The isolines are equidistant with $\Delta H=0.1$.

right corner. For the coupled model, this convection cell intrudes further into the flow domain than for the pseudo-coupled model. Again, this reflects the effect of buoyancy: intruding dense seawater tends to sink, which can only be captured by coupling. It is also worth noticing that $\alpha=1$ appears to mark the onset of this convection cell as the isolines for the coupled problem tend to become horizontal at the bottom of the sea boundary. Note that $H(\xi)$ for the pseudo-coupled model is exact at first order in $\alpha$ as discussed in the previous section. The first-order approximation for the coupled model, however, is not expected to be consistent for such values of $\alpha$, because it is strictly valid only for the weak coupling. This issue is discussed below.

Figure 3 demonstrates the accuracy of the first-order approximations of hydraulic head by comparing the head isolines obtained from the first-order approximation (4.13) of the coupled problem (solid lines) and from direct numerical simulations (dotted lines) of the full problem (2.20)-(2.23). SUTRA simulations of the pseudocoupled problem produce solutions for freshwater heads that are identical to the exact analytical solution (3.7), and are not displayed here.

For weak coupling $(\alpha=0.25$ and 0.5$)$, the head isolines obtained from the firstorder approximation coincide with the ones obtained from SUTRA, confirming the consistency and accuracy of our perturbation solution. As $\alpha$ increases, the first-order solution deviates from its numerical counterpart. For moderate coupling $(\alpha=1)$, the first-order approximation remains fairly robust. The largest deviations from the numerical solutions are observed in the lower right half of the flow domain, close to the saltwater boundary. This is because the boundary conditions at the bottom and top of the flow domain are expressed by (2.20) and (3.5b) in terms of the salt concentration 

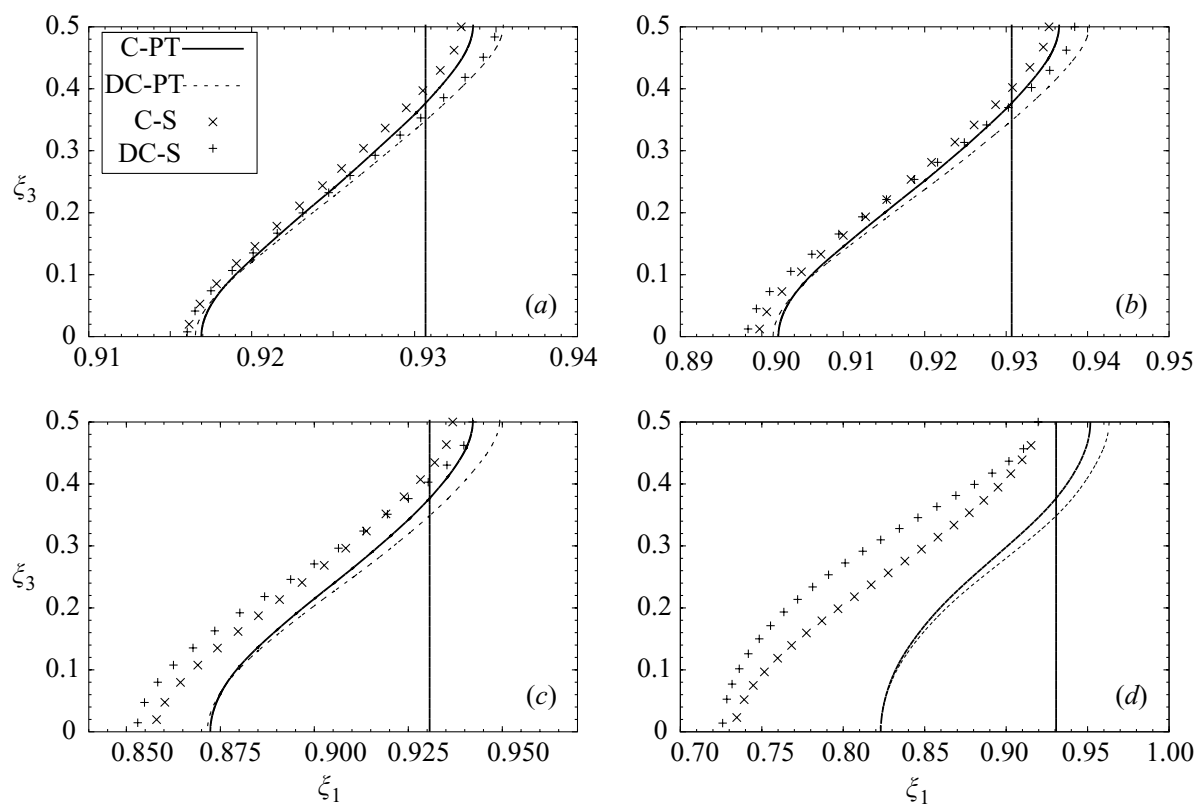

FIGURE 4. Isolines $C(\xi)=0.5$ for the salt concentration given by the first-order solutions (4.14) and (4.16) of the coupled (C-PT) and pseudo-coupled (DC-PT) problems, respectively, as well as by direct numerical simulations with SUTRA of the coupled (C-S) and pseudo-coupled (DC-S) problems. The following parameters are used: $\zeta=0.5, P e=10$, and $(a) \alpha=0.25$, (b) $\alpha=0.5$, (c) $\alpha=1.0$, and $(d) \alpha=2.0$. The vertical solid lines are the $C_{0}\left(\xi_{1}\right)=0.5$ isolines corresponding to the zeroth-order approximation ('bare solution') of $C(\xi)$.

$C(\boldsymbol{\xi})$. The first-order approximation of $C(\boldsymbol{\xi})$ is given by the 'bare' solution $C_{0}\left(\xi_{1}\right)$, which is independent of $\xi_{3}$. However, saltwater intrusion is most pronounced in the lower right corner, where a convection cell develops resulting in a strong dependence on $\xi_{3}$. This feature is a higher-order effect and cannot be accounted for by the first-order approximation. For strong coupling $(\alpha=2)$, the difference between the two solutions is significant and occurs throughout the flow domain $\Omega_{d}$. The isolines of the full numerical solution are more curved than those given by the first-order approximation, indicating larger vertical flow.

Of course, it should come as no surprise that the accuracy of the perturbation solution (4.13) deteriorates with increasing $\alpha$. Indeed, the use of $\alpha$ as a (small) perturbation parameter formally limits the range of applicability of (4.13) to $\alpha \ll 1$. Nevertheless, figure 3 demonstrates that the perturbation solution (4.13) for freshwater head $H(\xi)$ remains accurate for moderate coupling with $\alpha$ as high as 1 .

On a separate note, figure 3 shows that the extent of the convection cell increases with the degree of coupling between the flow and transport processes, i.e. with increasing coupling parameter $\alpha$, which is qualitatively consistent with the observations in figure 2, which compares the first-order approximations to the coupled and pseudo-coupled scenarios.

\subsection{Salt concentration}

Figure 4 illustrates the impact of the modelling assumptions by comparing the $C(\xi)=0.5$ isolines of the salt concentration $C(\xi)$ computed with the first-order approximations of the solutions to the coupled (4.14) and pseudo-coupled (4.16) problems, as well as with the corresponding direct numerical simulations (SUTRA). 

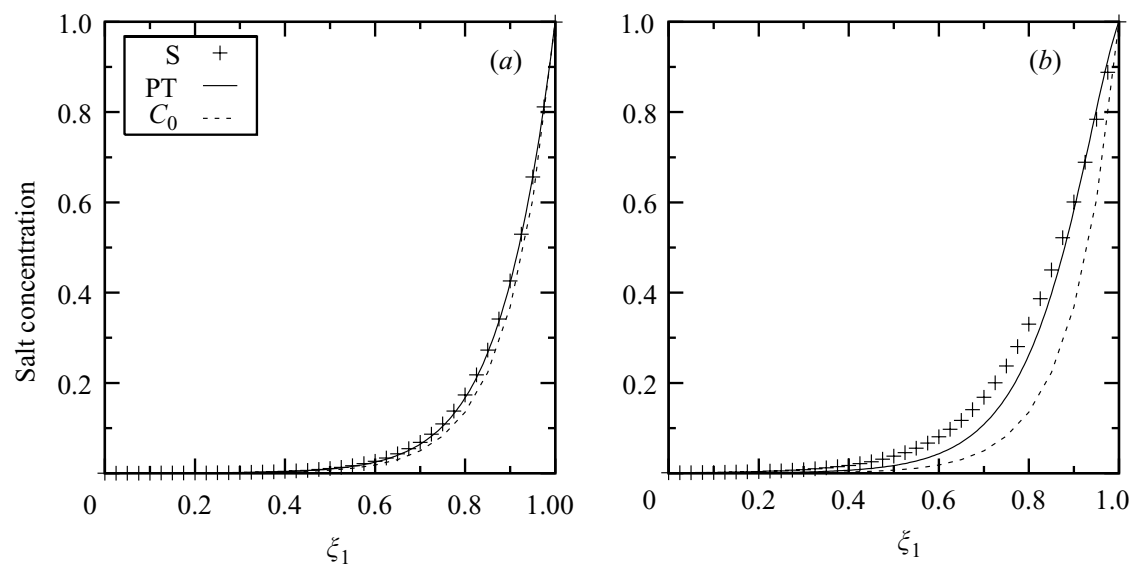

FIGURE 5. Salt concentration profiles at $\xi_{3}=0.1$ given by the first-order solution (4.14) (PT), direct numerical simulations with SUTRA (S), and the zeroth-order solution $C_{0}\left(\xi_{1}\right)$. The following parameters are used: $\zeta=0.5, P e=10$, and $(a) \alpha=0.25,(b) \alpha=1$.

All solutions qualitatively capture the main features of the phenomenon. Specifically, one can see how the heavier saltwater intrudes at the right bottom of the flow domain, while it is pushed back by the outflowing lighter freshwater at the right top.

For the full range of coupling $\alpha$, the difference between the coupled and pseudocoupled concentration isolines is significant. In the coupled model, saltwater intrudes consistently more at the top of the domain and less at the bottom. As saltwater intrudes, it loses energy due to diffusion of salt, a feature which is inherently connected to the coupled nature of flow and transport. Since this mechanism is not captured by the pseudo-coupled model, it overestimates the saltwater intrusion. Furthermore, as $\alpha$ increases, the intersection between the isoline and the top boundary moves seaward, reflecting the 'wash-out' effect of freshwater outflow, which concentrates in the top portion. This leads to the increase in the convexity of the concentration isolines predicted by the pseudo-coupled model. The differences between the coupled and pseudo-coupled models are not completely accounted for by the first-order approximations because, as discussed in $\S 4.3$, low order in $\alpha$ means low order with respect to the approximation of the transport solution for the pseudo-coupled model, while in the coupled model it implies low order with respect to the simultaneous approximation of the solutions for the flow and transport problems. The density effects leading to the above features are of higher order in $\alpha$.

Figure 4 also demonstrates the accuracy of the perturbation solutions to the coupled and pseudo-coupled problems. For all $\alpha$, the zeroth-order approximation of salt concentration $C_{0}\left(\xi_{1}\right)$ significantly underestimates the extent of seawater intrusion in the lower two thirds of the flow domain, and overestimates it in the upper part of the flow domain. For weak coupling $(\alpha=0.25$ and 0.5$)$, the first-order approximations of the coupled and pseudo-coupled solutions are in a good agreement with 'true' solutions obtained by SUTRA. The discrepancy between the first-order solutions and their numerical counterparts increases with $\alpha$, as should be expected from the perturbative nature of the former.

Figure 5 further elucidates the issue of accuracy of the first-order approximation by providing the concentration distributions along the horizontal cross-section $\xi_{3}=0.1$. For weak coupling $(\alpha=0.25)$, the first-order approximation of the solution to the 
coupled problem is practically indistinguishable from the solution obtained with direct numerical simulations (SUTRA). As expected, the difference between the two solutions becomes more pronounced as $\alpha$ increases.

Another important feature revealed by figure 5 is that saltwater intrudes further than would be predicted by the model based on diffusion of salt in uniform flow, i.e. by $C_{0}\left(\xi_{1}\right)$.

\section{Small Péclet numbers}

As defined by (2.17), small Péclet numbers $P e$ occur in scenarios characterized either by very large values of the diffusion coefficient $D$ or by very small values of the freshwater flux (i.e. either by very small hydraulic conductivity $K$ or by very small pressure drop $\Delta h_{0}$ ). Both situations can occur in practice: tidal fluctuations induce temporal fluctuations of the flow velocity, which lead to large effective dispersion in the upper portion of coastal aquifers (Dentz \& Carrera 2003); in diffusion experiments with variable-density flows in porous media (Dror, Yaron \& Berkowitz 2003), a narrow sample $(\zeta \approx 10)$ is placed between two reservoirs, one containing freshwater and the other saltwater, while keeping the mean pressure equilibrated on both sides. This implies $h_{0}=(1+\epsilon / 2) d$. These conditions are imposed to eliminate advection, i.e. to obtain a small Péclet number Pe. The resulting coupling parameter $\alpha$ and Péclet number $P e,(2.17)$, then are given by

$$
\alpha=\frac{2}{\zeta}, \quad P e=\frac{K \epsilon d}{\theta D},
$$

respectively. For a typical value of $\zeta=10, \alpha=1 / 5$, while the Péclet number is proportional to the (small) density contrast $\epsilon$. Nevertheless, in practice advection is observed for such a scenario (Poupeleer et al. 2003) and, in fact, it plays an important role as we will illustrate in the following.

This section is devoted to analysing the case of small Pe. Taking the limit of (2.22) as $P e \rightarrow 0$, we obtain a transport equation for the zeroth-order approximation of the salt concentration $C_{\mathrm{sp}}$ in the small-Péclet-number regime,

$$
\nabla^{2} C_{\mathrm{sp}}^{(0)}(\xi)=0 .
$$

A solution of (5.2) subject to the boundary conditions (2.23) is

$$
C_{\mathrm{sp}}^{(0)}(\xi)=C_{\mathrm{sp}}^{(0)}\left(\xi_{1}\right)=\xi_{1},
$$

which predicts that the salt concentration decreases linearly with distance from the seawater boundary. The corresponding flow problem is given by substituting (5.3) into (2.20)-(2.21),

$$
\nabla^{2} H_{\mathrm{sp}}^{(0)}(\xi)=0
$$

subject to the boundary conditions

$$
\begin{array}{cc}
H_{\mathrm{sp}}^{(0)}\left(0, \xi_{3}\right) & =1, \quad H_{\mathrm{sp}}^{(0)}\left(1, \xi_{3}\right)=\alpha\left(\zeta-\xi_{3}\right), \\
\frac{\partial H_{\mathrm{sp}}^{(0)}\left(\xi_{1}, 0\right)}{\partial \xi_{3}} & =-\alpha \xi_{1}, \quad \frac{\partial H_{\mathrm{sp}}^{(0)}\left(\xi_{1}, \zeta\right)}{\partial \xi_{3}}=-\alpha \xi_{1} .
\end{array}
$$

Its solution gives an expression for the dimensionless hydraulic head $H_{\mathrm{sp}}^{(0)}(\boldsymbol{\xi})$,

$$
H_{\mathrm{sp}}^{(0)}(\xi)=1-\xi_{1}+\alpha \xi_{1}\left(\zeta-\xi_{3}\right)
$$



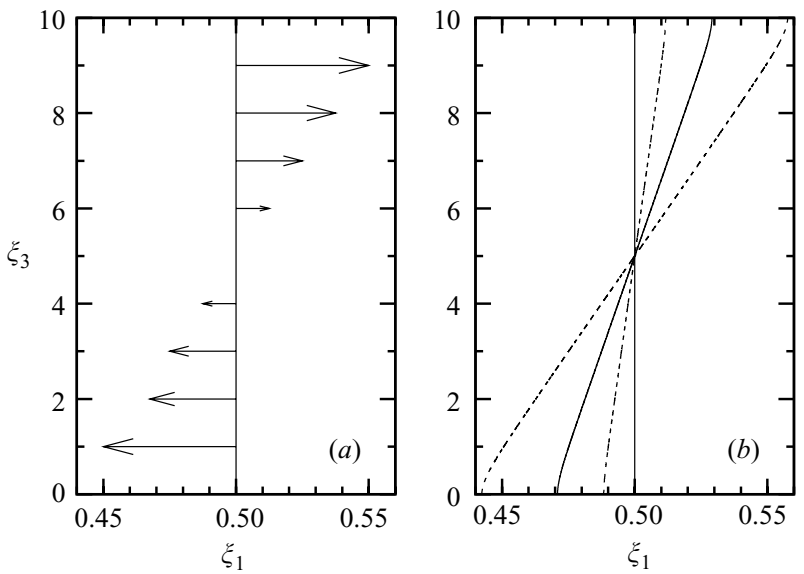

Figure 6. Solutions for small $P e$ with $\alpha=1 / 5$ and $\zeta=10:(a)$ the (linear) velocity profile; (b) the $C_{\mathrm{sp}}(\xi)=0.5$ isolines for $P e=0,0.1,0.25,0.5$ (at the top from left to right, respectively). For $P e=0$, this isoline represents the exact solution $C_{\mathrm{sp}}^{(0)}$; for the remaining Péclet numbers $P e$, it is computed with the first-order solution $C_{\mathrm{sp}}^{(0)}+C_{\mathrm{sp}}^{(1)}$. Note that only a narrow portion of the domain is represented.
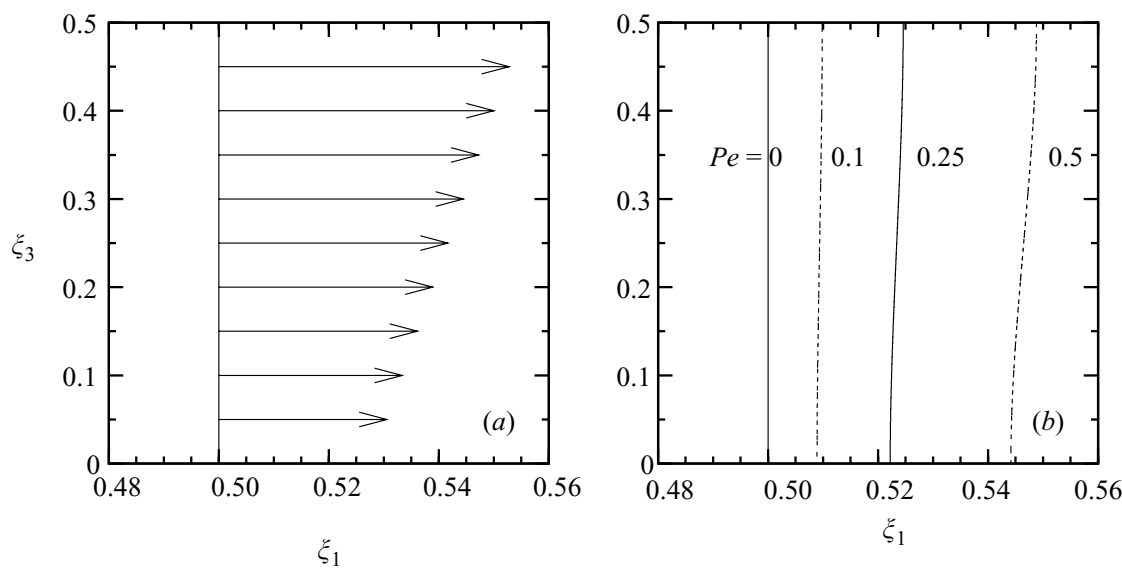

FIGURE 7. As figure 6 but with $\alpha=1, \zeta=1 / 2$.

According to (2.18), this yields the Darcy velocity

$$
U_{1}(\xi) \equiv U_{1}\left(\xi_{3}\right)=1+\alpha\left(\xi_{3}-\zeta\right), U_{3}(\xi) \equiv 0 .
$$

The result is a one-dimensional (horizontal) shear flow with a linear velocity profile, see figures $6(a)$ and $7(a)$. For $\alpha<\zeta^{-1}$ the flow velocity is positive, i.e. $v_{1}\left(\xi_{3}\right)>0$. For $\alpha \geqslant \zeta^{-1}$ the Darcy flow changes direction from seaward for $\left(\zeta-\alpha^{-1}\right)<\xi_{3} \leqslant \zeta$ to inland for $0 \leqslant \xi_{3}<\left(\zeta-\alpha^{-1}\right)$. In a typical diffusion experiment, $\zeta-\alpha^{-1}=\zeta / 2$. The velocity profile for the typical value of $\zeta=10$ is shown figure $6(a)$. It demonstrates that advection plays an important role, and that such an experimental setup does not eliminate advection.

For small but finite Péclet numbers $P e$, we obtain analytical solutions for salt concentration $C_{\mathrm{sp}}(\xi)$ and hydraulic head $H_{\mathrm{sp}}(\xi)$ via perturbation expansions in the 
powers of $P e$,

and

$$
C_{\mathrm{sp}}(\xi)=C_{\mathrm{sp}}^{(0)}\left(\xi_{1}\right)+\sum_{n=1}^{\infty} P e^{n} C_{\mathrm{sp}}^{(n)}(\xi)
$$

$$
H_{\mathrm{sp}}(\xi)=H_{\mathrm{sp}}^{(0)}\left(\xi_{3}\right)+\sum_{n=1}^{\infty} P e^{n} H_{\mathrm{sp}}^{(n)}(\xi)
$$

Here

$$
H_{\mathrm{sp}}^{(n)}(\xi)=\alpha \eta_{C}\left(\xi, C_{\mathrm{sp}}^{(n)}\right), \quad n \geqslant 1
$$

and $\eta_{C}$ is defined by $(3.5 a)$.

From (2.22), we obtain the integral equation for $C_{\mathrm{sp}}(\xi)$,

$$
C_{\mathrm{sp}}(\xi)=\xi_{1}+P e \int_{\Omega_{d}} g_{\mathrm{sp}}\left\{\nabla^{\prime} H_{\mathrm{sp}} \cdot \nabla^{\prime} C_{\mathrm{sp}}+\alpha C_{\mathrm{sp}} \frac{\partial C_{\mathrm{sp}}}{\partial \xi_{3}^{\prime}}\right\} \mathrm{d} \xi^{\prime},
$$

where the Green function $g_{\mathrm{sp}}$ is derived from the Green function (A 24) by taking the limit

$$
g_{\mathrm{sp}}\left(\xi, \xi^{\prime}\right) \equiv \lim _{P_{e} \rightarrow 0} \frac{g\left(\xi, \xi^{\prime}\right)}{P e}=G\left(\xi, \xi^{\prime}\right)
$$

and $G\left(\xi, \xi^{\prime}\right)$ is given by (A 10). Inserting (5.8) and (5.9) into (5.11) allows one to express $C_{\mathrm{sp}}^{(n)}$ in terms of $C_{\mathrm{sp}}^{(k)}$ with $k<n$. In particular, the first-order term $C_{\mathrm{sp}}^{(1)}$ is given by

$$
C_{\mathrm{sp}}^{(1)}(\xi)=\frac{(1-\alpha \zeta)}{2} \xi_{1}\left(\xi_{1}-1\right)-\alpha \int_{\Omega_{d}} G \xi_{3}^{\prime} \mathrm{d} \xi^{\prime}
$$

and the higher-order terms have the form

$$
C_{\mathrm{sp}}^{(n+1)}=\sum_{j=0}^{n} \int_{\Omega_{d}} G\left[\nabla^{\prime} H_{\mathrm{sp}}^{(j)} \nabla^{\prime} C_{\mathrm{sp}}^{(n-j)}+C_{\mathrm{sp}}^{(j)} \frac{\partial C_{\mathrm{sp}}^{(n-j)}}{\partial \xi_{3}^{\prime}}\right] \mathrm{d} \xi^{\prime} .
$$

Figures $6(b)$ and $7(b)$ show the $C_{\mathrm{sp}}(\xi)=0.5$ isoline for $P e=0,0.1,0.25$ and 0.5 . For $P e=0$, this isoline represents the exact solution $C_{\mathrm{sp}}^{(0)}$; for the remaining $P e$, it is computed with the first-order solution $C_{\mathrm{sp}}^{(0)}+C_{\mathrm{sp}}^{(1)}$. The isoline for $P e=0$ is a vertical line at $\xi_{1}=0.5$.

For $\zeta=10$ and $\alpha=1 / 5$, the 0.5 isolines are point-symmetric about $(0.5,5)$. For finite Péclet numbers, the salt concentration is pushed toward the saltwater boundary in the upper half of the domain and toward the freshwater boundary in the lower half following the linear velocity profile (5.7). The 0.5 isoline 'rotates' toward the saltwater boundary, and its slope increases with $P e$. The resulting concentration profiles are considerably different from the purely diffusive case.

For $\zeta=1 / 2$ and $\alpha=1$, the velocity profile is given in figure $7(a)$. The uniform flow velocity is directed toward the saltwater boundary with downward decreasing flow velocity. The resulting 0.5 isolines are shown in figure $7(b)$. As $P e$ increases, the concentration isolines are pushed toward the sea boundary, more so at the top than at the bottom of the domain. As expected, the deviation from the vertical straight line increases with $P e$. Yet the deviations from a purely diffusive concentration profile in this case are not as pronounced as in the scenario characterized by $\zeta=10$ and $\alpha=1 / 5$. 
These results clearly demonstrate that the diffusion experiments cannot be conducted with vertical samples of two fluids with different densities because (i) the Péclet number $P e=K \epsilon d /(2 \theta D)$ is small but finite, and more importantly, (ii) diffusion may become negligible compared to advection in view of the velocity profile shown in figure 6(a). Obviously, one can overcome these difficulties by setting the sample horizontally, as pointed out by Dror et al. (2003).

Finally, it is worth pointing out that the perturbation solutions for salt concentration (4.14) and hydraulic head (4.13) - obtained for the case of weak coupling ( $\operatorname{cmall} \alpha$ ) but intermediate $P e$ - converge to the exact solutions (5.3) and (5.6), respectively, in the limit as $P e \rightarrow 0$.

\section{Large Péclet numbers}

In the absence of diffusion there is no mixing between salt- and freshwater. The two fluids are separated by a sharp interface. The sharp-interface approximation for saltwater intrusion into coastal aquifers has been studied extensively (e.g. Bear \& Dagan 1964; Bear 1972; Kacimov \& Obnosov 2001; Kacimov 2001; Bakker, Oude Essink \& Langevin 2004). Within this modelling framework, the interface is treated as a free surface, whose position can be determined by, e.g., the hodograph method (e.g. Bear 1972), techniques based on the method of boundary value problems (e.g. Kacimov 2001).

The limit of $P e \rightarrow \infty$ represents a singular limiting case and leads to paradox phenomena. There is a jump discontinuity along the interface and, as a result, there is no freshwater outflow, since at the seaside boundary the salt concentration is $c=1$. This is not a problem for the case of finite diffusion (finite $P e$ ), because the salt concentration is a continuous function of space. Hence, analytical solutions to the sharp-interface problem usually disregard the seepage face, which leads to a vortexlike singularity at the upper right corner of the outflow boundary (e.g. de Josselin de Jong 1958; Bakker et al. 2004).

In the limit as $P e \rightarrow \infty$, the transport equation (2.19) changes its character from a second-order to a first-order partial differential equation,

$$
\boldsymbol{U}(\boldsymbol{\xi}) \cdot \nabla C_{\mathrm{si}}(\xi)=0,
$$

where the salt concetration in the sharp-interface approximation $C_{\mathrm{si}}=1$ behind the front and 0 ahead of the front; and the dimensionless flow velocity $\boldsymbol{U}(\boldsymbol{\xi})$ is given by (2.18). The divergence-free flow velocity can be expressed in terms of the stream function $\psi(\xi)$ by

$$
\boldsymbol{U}(\boldsymbol{\xi})=\left(-\frac{\partial}{\partial \xi_{3}}, \frac{\partial}{\partial \xi_{1}}\right)^{T} \psi(\xi)
$$

A solution $C_{\mathrm{si}}(\xi)$ of $(6.1)$ can be written as a function $F$ of $\psi$,

$$
C_{\text {si }}(\xi)=F[\psi(\xi)] \text {. }
$$

In the absence of molecular diffusion, the interface coincides with the $\psi(\xi)=\psi_{0}$ isoline on which $\boldsymbol{U}(\boldsymbol{\xi})=\mathbf{0}$, i.e.

$$
\left.\psi(\xi)\right|_{\boldsymbol{U}_{(\xi)=0}}=\psi_{0} .
$$

Hence, the solution of the salt transport equation (6.1) is the Heaviside function

$$
C_{\text {si }}(\xi)=\Theta\left[\psi(\xi)-\psi_{0}\right], \quad \xi_{1}<1,
$$

which defines the sharp interface between salt- and freshwater. 

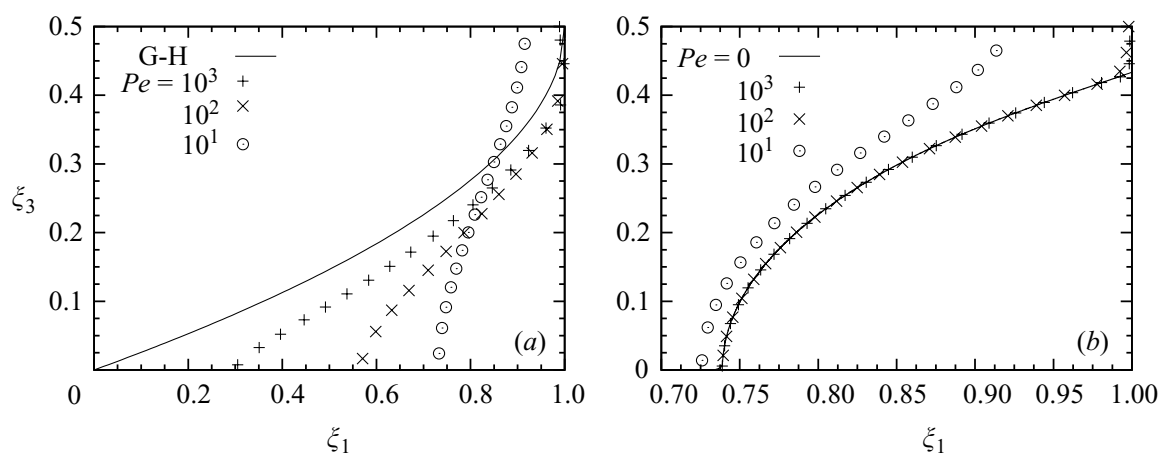

FIGURE 8. Concentration isolines $C(\xi)=0.5$ obtained with the direct numerical simulations (SUTRA) from $(a)$ the coupled Henry problem and $(b)$ the pseudo-coupled model. The solid line in $(a)$ shows the sharp interface given by the Ghyben-Herzberg approximation (6.6), while the solid line in $(b)$ shows the sharp interface given by (6.5). The following parameters are used: $\alpha=2, \zeta=1 / 2$, and $P e=10,10^{2}$ and $10^{3}$.

Figure 8(a) shows the 0.5 concentration isolines computed with direct numerical simulations (SUTRA) for $\alpha=2$ and $P e=10,10^{2}$ and $10^{3}$. As Péclet number $P e$ increases, the 'toe' (the point defined as the intersection of the $C(\xi)=0.5$ isoline with the $\xi_{1}$-axis) moves toward the freshwater boundary, while the 0.5 isoline is pushed toward the sea boundary at the top of the domain. In the diffusion-dominated transport scenario studied in the previous section, the 0.5 isoline moves backward with increasing $P e$. In the advection-dominated scenario considered in this section, we observe the opposite behaviour. This is due to the fact that now saltwater intrusion is dominated by the flow pattern, and the position of the mixing zone, or the sharp interface in the $P e=\infty$ limit, coincides with a streamline of the flow field. Intruding saltwater loses energy due to dilution. As $P e$ increases, the dilution of salt is reduced, which results in the inland movement of the toe. Note the existence of a seepage face at the upper seaside boundary for increasing $P e$.

The solid line in figure $8(a)$ represents the position of the sharp interface predicted by the Ghyben-Herzberg approximation, which assumes that saltwater is immobile (e.g. Bear 1972). Using the condition of pressure equilibrium along the interface between fresh- and seawater and the conservation of total flux, one obtains the Ghyben-Herzberg approximation for the interface position $z\left(\xi_{1}\right)$,

$$
z\left(\xi_{1}\right)=\zeta-\sqrt{\zeta^{2}-\frac{2 Q}{\alpha}\left(\xi_{1}-\xi_{\text {toe }}\right)} .
$$

Here the position of the toe, $\xi_{\text {toe }}$, is given by

$$
\xi_{\text {toe }}=1-\frac{\zeta^{2} \alpha}{2 Q}
$$

in terms of the total flux $Q$ through the flow domain. We derive an exact solution for the flux,

$$
Q=-\int_{0}^{\zeta} \frac{\partial H(\xi)}{\partial \xi_{1}} \mathrm{~d} \xi=\zeta\left(1-\frac{\alpha \zeta}{2}\right)
$$

which is valid for an arbitrary Péclet number.

Figure 8(a) demonstrates that the sharp-interface approximation overestimates the position of the toe for large but finite $P e$. The seepage face is not represented in this 
approximation and the outflow of freshwater concentrates at the upper right corner, which becomes a singular point.

\subsection{Pseudo-coupled model for large Pe}

The pseudo-coupled Henry problem described in $\S 3.1$ is exactly solvable in the limit of $P e \rightarrow \infty$. The stream function $\psi(\xi)$ for $\boldsymbol{U}(\boldsymbol{\xi})$ corresponding to the exact solution (3.7) for the hydraulic head $H_{\mathrm{pc}}(\boldsymbol{\xi})$ is given by

$$
\psi_{\mathrm{pc}}(\xi)=1-\xi_{3}+\frac{\xi_{3} \zeta}{2}+\sum_{l=1}^{\infty} a_{l} \sin \left(\frac{l \pi \xi_{3}}{\zeta}\right) \frac{\cosh \left(l \pi \xi_{1} / \zeta\right)}{\sinh (l \pi / \zeta)}
$$

Substituting (6.9) into (6.5), we obtain the exact solution for salt concentration $\left.C_{(} \boldsymbol{\xi}\right)$.

Figure $8(b)$ compares the position of the sharp interface determined from this solution with its counterpart computed with direct numerical simulations (SUTRA) of the pseudo-coupled Henry problem with $P e=10,10^{2}$ and $10^{3}$. The exact sharpinterface solution is in good agreement with its numerical counterpart for large Péclet numbers $\left(P e=10^{3}\right.$ and $\left.10^{2}\right)$. Not surprisingly, this agreement deteriorates as $P e$ becomes smaller.

A key feature of the pseudo-coupled model is the absence of the vortex-type singularity at the outflow boundary and the seepage face. This feature is consistent with other sharp-interface scenarios (e.g. Kacimov 2001), and results in the boundary segment along which freshwater discharges into the ocean.

Comparison of figures $8(a)$ and $8(b)$ shows the remarkable qualitative and quantitative differences between the coupled and pseudo-coupled formulations for large $P e$. Thus, flow and salt transport are more sensitive to density variation in the advection-dominated case (large Péclet numbers $P e$ ). For intermediate $P e$, flow and salt transport are described reasonably well by the much simpler pseudo-coupled problem.

\section{Summary and conclusions}

We analysed the Henry problem for steady-state variable-density flow and transport in homogeneous porous media. This setup is often used to represent seawater intrusion in coastal aquifers, wherein fresh water is discharged into the sea, while salt water is advected and diffused into the aquifer. In this process, a transition zone between fresh and saline water develops, thus endangering the quality of fresh groundwater. The coupling of flow and transport phenomena is caused by the dependence of the water density on the salt concentration.

The dimensionless form of the governing equations shows that flow and transport processes are effectively governed by two dimensionless groups. These are the Péclet number $P e$ and the coupling parameter $\alpha$. The former quantifies the relative effects of advection and diffusion, while latter quantifies the relative importance of density effects and external forces (boundary conditions). Specifically, the coupling parameter $\alpha$ measures the strength of the coupling between fluid flow and salt movement, and quantifies the deviation of the flow velocity driven by the seaside boundary condition from a uniform flow condition.

In conjunction with the fully coupled Henry problem, we consider its pseudocoupled formulation, which assumes that coupling of flow and transport is only due to the saltwater boundary condition at the seaside, while neglecting effects of density variations within the domain. One of the objectives of this study was to establish the accuracy and the validity of this simplified model for the full range of 
the Péclet number $P e$. Another was to analyse the relative effects of various transport mechanisms, as well as the strength of coupling between flow and transport.

For intermediate values of the Péclet number $P e$, we used a perturbation expansion in the (small) coupling parameter $\alpha$ to derive analytical solutions for the spatial distributions of the water potential (hydraulic head) and the salt concentration. The salient feature of these solutions is that the flow and transport equations are decoupled at every approximation order. The first-order approximations of the analytical solutions for the water potential and the salt concentration were compared with direct numerical simulations (SUTRA). We found the following.

(i) The first-order approximation of hydraulic head is accurate for moderate density coupling $(\alpha<1)$. For stronger coupling between flow and salt transport (larger $\alpha$ ), higher-order contributions of the perturbation series gain importance and lead to the loss of accuracy of the perturbation solutions.

(ii) The coupled and pseudo-coupled models results in similar flow patterns when $\alpha \leqslant 1$.

(iii) The coupled solution for salt concentration has a conceptual advantage over its pseudo-coupled counterpart, in that it retains the information about energy losses linked to the diffusion of salt.

(iv) The pseudo-coupled formulation of the Henry problem overestimates the volume of aquifer contaminated by the salt.

For small Péclet numbers $P e$ (the diffusion-dominated seawater intrusion), we used a perturbation expansion in $P e$ to derive approximate solutions for hydraulic head and salt concentration. This regime is typically used in experimental studies of diffusion in variable density fluids, and may occur in coastal aquifers. We found the following.

(i) For $P e=0$, the salt concentration decreases linearly toward the freshwater boundary while the corresponding flow field is given by a linear shear flow.

(ii) Even for very small Péclet numbers, advective transport may dominate diffusive transport as a result of the linear velocity profile.

(iii) As $\mathrm{Pe}$ increases, the concentration isolines deviate from the linear behaviour and move toward the seawater boundary. This behaviour is in contrast to that observed in the limiting case of very large $P e$, where saltwater intrudes more for decreasing $P e$ as a consequence of less energy loss due to the dilution of salt.

For large Péclet numbers $P e$, a clearly defined mixing zone develops, with the (singular) limit of $P e \rightarrow \infty$ corresponding to a sharp interface. We analysed the accuracy of the Ghyben-Herzberg approximation by comparing to the direct numerical simulations. We also developed an analytical solution of the pseudocoupled model in the limit of $P e \rightarrow \infty$. We found the following.

(i) Both the coupled and pseudo-coupled formulations of the Henry problem capture the presence of a seepage face along the seaside boundary. This salient feature of seawater intrusion is absent in most sharp-interface models.

(ii) Flow and salt transport are more sensitive to density variation in the advectiondominated case (large Péclet numbers $P e$ ).

(iii) For intermediate $P e$, flow and salt transport are described reasonably well by the much simpler pseudo-coupled problem.

The perturbation formalisms developed provide a systematic methodological approach and an effective analytical tool for the analysis of stable densitydependent flow problems in homogeneous porous environments. The general methodology introduced can be applied to a range of boundary value problems, including temperature- or pressure-driven environmental density flows occurring in deep aquifers, and is not restricted to the particular conditions considered here. 
Furthermore, it can be straightforwardly extended to include time-dependent flow and transport scenarios as well as to density-dependent flow and transport in heterogeneous environments. As such, the analytical method presented can serve as the basis for a systematic investigation of the influence of ever present small-scale medium heterogeneities on effective large-scale flow and transport.

The work of D.T. was performed at Los Alamos National Laboratory (LA-UR 045497) under the auspices of the National Nuclear Security Agency of US Department of Energy under contract W-7405-ENG-36. This work was supported in part by the US Department of Energy under the DOE/BES Program in the Applied Mathematical Sciences, Contract KC-07-01-01, and in part by the LDRD Program at Los Alamos National Laboratory. M.D. gratefully acknowledges the financial support of the Spanish Secretaría de Estado de Educación y Universidades and the program 'Ramon $y$ Cajal' of the Spanish Ministry of Science and Technology. The authors thank the Departament d'Universitat, Recerca i Societat de la Informació of the Catalan government for financial support.

\section{Appendix. Green functions}

In the following we derive the Green functions for the flow problem in $\S 3$, and for the integral formulation of the the transport problem in $\S 4.1$.

\section{A.1. Green functions for the flow problem}

The Green function $G\left(\boldsymbol{\xi}, \boldsymbol{\xi}^{\prime}\right)$ for (2.20)-(2.21) can be obtained by taking the limit of the Green function for the corresponding time-dependent diffusion problem (Carslaw \& Jaeger 1959),

$$
\begin{aligned}
G\left(\boldsymbol{\xi}, \boldsymbol{\xi}^{\prime}\right) & =\frac{2}{\zeta \pi^{2}} \sum_{m=1}^{\infty} \frac{1}{m^{2}} \sin \left(m \pi \xi_{1}\right) \sin \left(m \pi \xi_{1}^{\prime}\right) \\
+ & \frac{4 \zeta}{\pi^{2}} \sum_{m, n=1}^{\infty} \frac{1}{m^{2} \zeta^{2}+n^{2}} \sin \left(m \pi \xi_{1}\right) \sin \left(m \pi \xi_{1}^{\prime}\right) \cos \left(n \pi \frac{\xi_{3}}{\zeta}\right) \cos \left(n \pi \frac{\xi_{3}^{\prime}}{\zeta}\right)
\end{aligned}
$$

This expression can be simplified by summing the first sum on the right-hand side and one of the double sums by using the relations

$$
\begin{aligned}
2 \cos \left(n \pi \frac{\xi_{3}}{\zeta}\right) \cos \left(n \pi \frac{\xi_{3}^{\prime}}{\zeta}\right) & =\cos \left(n \pi \frac{\xi_{3}+\xi_{3}^{\prime}}{\zeta}\right)+\cos \left(n \pi \frac{\xi_{3}-\xi_{3}^{\prime}}{\zeta}\right), \\
2 \sin \left(m \pi \xi_{1}\right) \sin \left(m \pi \xi_{1}\right) & =\cos \left[m \pi\left(\xi_{1}-\xi_{1}^{\prime}\right)\right]-\cos \left[m \pi\left(\xi_{1}+\xi_{1}^{\prime}\right)\right]
\end{aligned}
$$

and (Gradshteyn \& Ryzhik 1980)

$$
\begin{aligned}
\sum_{n=1}^{\infty} \frac{1}{n^{2}+\gamma^{2}} \cos \left(n \pi \frac{\xi}{b}\right) & =\frac{\pi}{2 \gamma} \frac{\cosh \left[\gamma \pi\left(1-\frac{\xi}{b}\right)\right]}{\sinh (\gamma \pi)}-\frac{1}{2 \gamma^{2}}, \\
\sum_{m=1}^{\infty} \frac{1}{m^{2}} \cos \left(m \pi \frac{\xi}{a}\right) & =\frac{\pi^{2}}{6}-\frac{\pi^{2}|\xi|}{2 a}+\frac{\pi^{2} \xi^{2}}{4 a^{2}} .
\end{aligned}
$$

Applying (A 2) and (A 4) to the second term on the right-hand side of (A 1) and defining $\gamma \equiv m \zeta$, we obtain 


$$
\begin{aligned}
& \sum_{m=1}^{\infty} \sin \left(m \pi \xi_{1}\right) \sin \left(m \pi \xi_{1}\right) \\
& \quad \times\left\{\frac{\cosh \left\{m \pi\left[\zeta-\left(\xi_{3}+\xi_{3}^{\prime}\right)\right]\right\}+\cosh \left[m \pi\left(\zeta-\left|\xi_{3}-\xi_{3}^{\prime}\right|\right)\right]}{\pi m \sinh (m \pi \zeta)}-\frac{2}{\zeta \pi^{2} m^{2}}\right\} .
\end{aligned}
$$

The third term on the right-hand side of (A A 6) cancels with the first term on the right-hand side of (A 1). For $\xi_{3}^{\prime}<\xi_{3}$, this yields

$$
G\left(\boldsymbol{\xi}, \boldsymbol{\xi}^{\prime}\right)=\frac{2}{\pi} \sum_{m=1}^{\infty} \sin \left(m \pi \xi_{1}\right) \sin \left(m \pi \xi_{1}^{\prime}\right) \cosh \left(m \pi \xi_{3}^{\prime}\right) \frac{\cosh \left[m \pi\left(\zeta-\xi_{3}\right)\right]}{m \sinh (m \pi \zeta)} .
$$

For $\xi_{3}^{\prime}>\xi_{3}$ the arguments $\xi_{3}$ and $\xi_{3}^{\prime}$ in (A 7) are switched.

To derive an alternative representation for the Green function $G\left(\xi, \xi^{\prime}\right)$, we consider the first and second sums on the right-hand side of (A 1) separately. Applying (A 3) and (A 5) to the first sum on the right-hand side of (A 1) gives

$$
\frac{2}{\zeta \pi^{2}} \sum_{m=1}^{\infty} \frac{1}{m^{2}} \sin \left(m \pi \xi_{1}\right) \sin \left(m \pi \xi_{1}\right)=\frac{\left(\xi_{1}+\xi_{1}^{\prime}\right)-\left|\xi_{1}-\xi_{1}^{\prime}\right|}{2 \zeta}-\frac{\xi_{1} \xi_{1}^{\prime}}{\zeta} .
$$

Applying (A 3) and (A 4) to the second term on the right-hand side of (A 1) and defining $\gamma \equiv n / \zeta$ yields

$$
\begin{aligned}
& \sum_{n=1}^{\infty} \cos \left(n \pi \frac{\xi_{3}}{\zeta}\right) \cos \left(n \pi \frac{\xi_{3}^{\prime}}{\zeta}\right) \\
\times & \frac{1}{\pi n \sinh (n \pi / \zeta)}\left\{\cosh \left[n \pi\left(\frac{1}{\zeta}-\frac{\left|\xi_{1}-\xi_{1}^{\prime}\right|}{\zeta}\right)\right]-\cosh \left[n \pi\left(\frac{1}{\zeta}-\frac{\xi_{1}+\xi_{1}^{\prime}}{\zeta}\right)\right]\right\} .
\end{aligned}
$$

Combining (A 8) and (A 9) leads to

$$
\begin{aligned}
G\left(\boldsymbol{\xi}, \boldsymbol{\xi}^{\prime}\right)=\frac{\xi_{1}^{\prime}}{\zeta}\left(1-\xi_{1}\right)+\frac{2}{\pi} \sum_{n=1}^{\infty} \cos & \left(n \pi \frac{\xi_{3}}{\zeta}\right) \cos \left(n \pi \frac{\xi_{3}^{\prime}}{\zeta}\right) \\
& \times \sinh \left(\frac{n \pi \xi_{1}^{\prime}}{\zeta}\right) \frac{\sinh \left[(n \pi / \zeta)\left(1-\xi_{1}\right)\right]}{n \sinh (n \pi / \zeta)}
\end{aligned}
$$

for $\xi_{1}^{\prime}<\xi_{1}$. For $\xi_{1}^{\prime}>\xi_{1}$ the arguments $\xi_{1}$ and $\xi_{1}^{\prime}$ in (A 10) are switched.

\section{A.2. Green function for the transport problem}

Following Morse \& Feshbach (1953), we replace (4.2) with

$$
\nabla^{2} \varphi(\xi)-P e \frac{\partial \varphi(\xi)}{\partial \xi_{1}}=-P e \rho(\xi)
$$

and set

$$
\begin{aligned}
& \varphi(\boldsymbol{\xi})=\sum_{m=1}^{\infty} F_{m}\left(\xi_{1}\right) \cos \left(m \pi \frac{\xi_{3}}{\zeta}\right)+\frac{F_{0}\left(\xi_{1}\right)}{2}, \\
& \rho(\xi)=\sum_{m=1}^{\infty} \rho_{m}\left(\xi_{1}\right) \cos \left(m \pi \frac{\xi_{3}}{\zeta}\right)+\frac{\rho_{0}\left(\xi_{1}\right)}{2},
\end{aligned}
$$

where the $F_{m}\left(\xi_{1}\right)$ are as yet unknown functions, and

$$
\rho_{m}\left(\xi_{1}\right)=\frac{2}{\zeta} \int_{0}^{\zeta} \rho(\xi) \cos \left(m \pi \frac{\xi_{3}}{\zeta}\right) \mathrm{d} \xi_{3} .
$$


Substituting (A 12) and (A 13) into (A 11) yields

$$
\frac{\mathrm{d}^{2} F_{m}}{\mathrm{~d} \xi_{1}^{2}}-\left(\frac{m \pi}{\zeta}\right)^{2} F_{m}-P e \frac{\mathrm{d} F_{m}}{\mathrm{~d} \xi_{1}}=-P e \rho_{m}\left(\xi_{1}\right)
$$

A solution of (A 15) is given by

$$
F_{m}\left(\xi_{1}\right)=y_{1}\left(\xi_{1}\right)\left[c_{1}+\int \frac{\rho_{m}\left(\xi_{1}^{\prime}\right) y_{2}\left(\xi_{1}^{\prime}\right) P e}{\Delta\left(y_{1}, y_{2}\right)} \mathrm{d} \xi_{1}^{\prime}\right]+y_{2}\left(\xi_{1}\right)\left[c_{2}-\int \frac{\rho_{m}\left(\xi_{1}^{\prime}\right) y_{1}\left(\xi_{1}^{\prime}\right) P e}{\Delta\left(y_{1}, y_{2}\right)} \mathrm{d} \xi_{1}^{\prime}\right]
$$

where the constants of integration $c_{1}$ and $c_{2}$ are determined from the boundary conditions, and $\Delta\left(y_{1}, y_{2}\right)$ is the Wronski determinant for two independent solutions of the homogeneous problem (A 15). Such solutions are

$$
\begin{aligned}
y_{1}\left(\xi_{1}\right) & =\exp \left(\frac{\xi_{1} P e}{2}\right) \sinh \left(B_{m} \xi_{1}\right) \\
y_{2}\left(\xi_{1}\right) & =\exp \left[-\frac{\left(1-\xi_{1}\right) P e}{2}\right] \sinh \left[B_{m}\left(1-\xi_{1}\right)\right] \\
B_{m} & \equiv \sqrt{\frac{P e^{2}}{4}+\left(\frac{m \pi}{\zeta}\right)^{2}},
\end{aligned}
$$

and the corresponding Wronski determinant is

$$
\Delta\left(y_{1}, y_{2}\right)=-\exp \left(\frac{\xi_{1} P e}{2}\right) \exp \left[-\frac{\left(1-\xi_{1}\right) P e}{2}\right] B_{m} \sinh \left(B_{m}\right) .
$$

Substituting (A 17), (A 18) and (A 20) into (A 16) and adjusting the limits of integration to satisfy the boundary conditions, we obtain

$$
F_{m}\left(\xi_{1}\right)=\int_{0}^{1} \rho_{m}\left(\xi_{1}^{\prime}\right) g_{m}\left(\xi_{1}, \xi_{1}^{\prime}\right) \mathrm{d} \xi_{1}^{\prime}
$$

where

$$
g_{m}\left(\xi_{1}, \xi_{1}^{\prime}\right)=\frac{P e \exp \left[\frac{\left(\xi_{1}-\xi_{1}^{\prime}\right) P e}{2}\right]}{B_{m} \sinh \left(B_{m}\right)} \begin{cases}\sinh \left(B_{m} \xi_{1}\right) \sinh \left(B_{m}\left(1-\xi_{1}^{\prime}\right)\right), & \xi_{1}^{\prime}>\xi_{1}, \\ \sinh \left(B_{m} \xi_{1}^{\prime}\right) \sinh \left(B_{m}\left(1-\xi_{1}\right)\right), & \xi_{1}>\xi_{1}^{\prime} .\end{cases}
$$

Substituting (A 13), (A 14) and (A 21) into (A 12) leads to

$$
\begin{aligned}
\varphi(\xi) & =\int_{0}^{1} \mathrm{~d} \xi_{1}^{\prime} \int_{0}^{\zeta} \rho\left(\xi^{\prime}\right) \frac{2}{\zeta} \sum_{m=0}^{\infty} c_{m} g_{m}\left(\xi_{1}, \xi_{1}^{\prime}\right) \cos \left(m \pi \frac{\xi_{3}}{\zeta}\right) \cos \left(m \pi \frac{\xi_{3}^{\prime}}{\zeta}\right) \mathrm{d} \xi_{3}^{\prime} \\
& =\int_{0}^{1} \mathrm{~d} \xi_{1}^{\prime} \int_{0}^{\zeta} \rho\left(\xi^{\prime}\right) g\left(\boldsymbol{\xi}, \boldsymbol{\xi}^{\prime}\right) \mathrm{d} \xi_{3}^{\prime},
\end{aligned}
$$

where $c_{m}=1 / 2$ for $m=0$ and $c_{m}=1$ else. Thus, for $\xi_{1}^{\prime}<\xi_{1}$, the Green function $g\left(\boldsymbol{\xi}, \boldsymbol{\xi}^{\prime}\right)$ is given by

$$
\begin{aligned}
g\left(\boldsymbol{\xi}, \boldsymbol{\xi}^{\prime}\right)= & \exp \left[\frac{\left(\xi_{1}-\xi_{1}^{\prime}\right) P e}{2}\right] \frac{2 P e}{\zeta} \sum_{m=0}^{\infty} c_{m} \cos \left(m \pi \frac{\xi_{3}}{\zeta}\right) \cos \left(m \pi \frac{\xi_{3}^{\prime}}{\zeta}\right) \\
& \times \sinh \left(B_{m} \xi_{1}^{\prime}\right) \frac{\sinh \left[B_{m}\left(1-\xi_{1}\right)\right]}{B_{m} \sinh \left(B_{m}\right)}
\end{aligned}
$$


where $c_{0}=1 / 2, c_{m}=1(m>0)$, and

$$
B_{m}=\sqrt{\frac{P e^{2}}{4}+\left(\frac{m \pi}{\zeta}\right)^{2}} .
$$

For $\xi_{1}^{\prime}>\xi_{1}$, the Green function $g\left(\xi, \xi^{\prime}\right)$ is obtained from (A 24) by switching the arguments $\xi_{1}^{\prime}$ and $\xi_{1}$.

\section{REFERENCES}

Abarca, E., Carrera, J., Held, R., Sánchez-Vila, X., Dentz, M., Kinzelbach, W. \& VázquezSUÑ́, E. 2005 Effective dispersion in seawater intrusion through heterogeneous aquifers. In Groundwater and Saline Intrusion (ed. L. Araguás, C. E. \& M. Manzano). Hidrogeología y Aguas Subterráneas, vol. 15, pp. 49-62. Instituto Geológico y Minero de España.

Aliewi, A. S., Mackay, R., Jayyousi, A., Nasereddin, K., Mushtaha, A. \& Yaqubi, A. 2001 Numerical simulation of the movement of saltwater under skimming and scavenger pumping in the Pleistocene aquifer of Gaza and Jericho areas, Palestine. Transp. Porous Media 43 (1), 195-212.

Alkalali, A. \& Rostron, B. 2003 Basin-scale analysis of variable-density groundwater flow: Nisku Aquifer, Western Canadian Sedimentary Basin. J. Geochemi. Explora. 78 (9), 313-316.

Bakker, M., Oude Essink, G. H. P. \& Langevin, C. D. 2004 The rotating movement of three immiscible fluids - a benchmark problem. J. Hydrology 287, 270-278.

Bear, J. 1972 Dynamics of Fluids in Porous Media. Elsevier.

Bear, J., Cheng, A. H.-D., Sorek, S., Ouazar, D. \& Herrera, I. (Eds.) 1999 Seawater Intrusion in Coastal Aquifers - Concepts, Methods, and Practices. Kluwer.

Bear, J. \& Dagan, G. 1964 Some exact solutions of interface problems by means of the hodograph method. J. Geophys. Res. 69, 1563-1572.

ButkovskiI, A. G. 1982 Green's Functions and Transfer Functions Bandbook. Halsted.

Carslaw, H. S. \& Jaeger, J. C. 1959 Conduction of Heat in Solids, 2nd edn. Oxford University Press.

Croucher, A. E. \& O'Sullivan, M. J. 1995 The Henry problem for saltwater intrusion. Water Resour. Res. 31 (7), 1809-1814.

Custodio, E., Bruggeman, G. A. \& Cotecchia, V. 1987 Groundwater Problems in Coastal Areas. Stud. and Rep. in Hydrol., vol. 35. UNESCO.

Dentz, M. \& CARrera, J. 2003 Effective dispersion in temporally fluctuating flow through a heterogeneous medium. Phys. Rev. E 036310.

Diersch, H. G. \& Kolditz, O. 2002 Variable-density flow and transport in porous media: approaches and challenges. Adv. Water Resour. 25, 899-944.

Dror, I., Yaron, B. \& Berkowitz, B. 2003 Response to comment on "Salt-pump mechanism for contaminant intrusion into coastal aquifers". Science 302 (5646).

Galeati, G., Gambolati, G. \& Neuman, S. P. 1992 Coupled and partially coupled EulerianLagrangian model of fresh-water-seawater mixing. Water Resour. Res. 28 (1), 149-165.

Gradshteyn, I. S. \& Ryzhik, I. M. 1980 Table of Integrals, Series, and Products. Academic.

Henry, H. R. 1964 Effects of dispersion on salt encroachment in coastal aquifers. Water Supply Pap. 1613-C. US Geol. Surv.

Herbert, A. W., Jackson, C. P. \& Lever, D. A. 1988 Coupled groundwater flow and solute transport with fluid density strongly dependent upon concentration. Water Resour. Res. 24 (10), 17811795.

Holzbecher, M. E. 1998 Modeling Density-Driven Flow in Porous Media. Springer.

Huppert, H. E. \& Woods, A. W. 1995 Gravity-driven flows in porous layers. J. Fluid Mech. 292, $55-69$.

DE Josselin DE JONG, G. 1958 Singularity distributions for the analysis of multiple fluid flow through porous media. J. Geophys. Res. 65, 3739-3758.

Kacimov, A. R. 2001 Analytical solution to a sharp interface problem in a vortex-generated flow. Water Resour. Res. 37, 3387-3391.

Kacimov, A. R. \& Obnosov, Y. V. 2001 Analytical solution for a sharp interface problem in sea water intrusion into a coastal aquifer. Proc. R. Soc. Lond. A 457, 3023-3038. 
Manickam, O. \& Homsy, G. M. 1995 Fingering instabilities in vertical miscible displacement flows in porous media. J. Fluid Mech. 288, 75-102.

Morse, P. M. \& Feshbach, H. 1953 Methods of Theoretical Physics. McGraw Hill.

Naji, A., Cheng, A. H.-D. \& Ouazar, D. 1998 Analytical stochastic solutions of saltwater/ freshwater interface in coastal aquifers. Stoch. Hydrol. Hydraul. 12, 413-429.

Oltean, C., Felder, C., Panfilov, M. \& Bues, M. A. 2004 Transport with a very low density contrast in Hele-Shaw cell and porous medium: Evolution of the mixing zone. Transp. Porous Media 55 (3), 339-360.

OPHORI, D. U. 2004 A simulation of large-scale groundwater flow and travel time in a fractured rock environment for waste disposal purposes. Hydrogeol. Proc. 18 (9), 1579-1593.

Panday, S., Huyakorn, P. S., Robertson, J. B. \& McGurk, B. 1993 A density-dependent flow and transport analysis of the effects of groundwater development in a fresh-water lens of limited areal extent - The Geneva area (Florida, USA) case study. J. Contaminant Hydrol. 12 (4), 329-354.

Paniconi, C., Khlaifi, I., Lecca, G., Giacomelli, A. \& Tarhouni, J. 2001 Modeling and analysis of seawater intrusion in the coastal aquifer of eastern Cap-Bon, Tunisia. Transp. Porous Media 43 (1), 3-28.

Pinder, G. F. \& COOPER, H. H. 1970 A numerical technique for calculating transient position of saltwater front. Water Resour. Res. 6 (3), 875-882.

Poupeleer, A. S., Carmeliet, J., Roels, S. \& Van Gemert, D. 2003 Validation of the salt diffusion coefficient in porous materials. Intl J. for Restoration Buildings Monuments 9 (6), 663-682.

Press, W. H., Teukolsky, S. A., Vetterling, W. T. \& Flannery, B. P. 1992 Numerical Recipes in $C, 2$ nd Edn. Cambridge University Press.

Schelkes, K., Vogel, P. \& Klinge, H. 2001 Density-dependent groundwater movement in sediments overlying salt domes. The Gorleben site example. Phys. Chem. Earth B Hydrol. Oceans Atmos. 26 (4), 361-365.

SCHINCARIOL, R. A. \& Schwartz, F. W. 1990 An experimental investigation of variable density flow and mixing in homogeneous and heterogeneous media. Water Resour. Res. 26 (10), 2317-2329.

Segol, G. 1994 Classic Groundwater Simulations - Proving and Improving Numerical Models. Prentice Hall.

Segol, G., Pinder, G. F. \& Gray, W. G. 1975 Galerkin-finite element technique for calculating transient position of saltwater front. Water Resour. Res. 11 (2), 343-347.

Simmons, C. T., Narayan, K. A., Woods, J. A. \& Herczeg, A. L. 2002 Groundwater flow and solute transport at the Mourquong saline-water disposal basin, Murray Basin, southeastern Australia. Hydrogeol. J. 10 (2), 278-295.

Simpson, M. J. \& Clement, T. P. 2003 Theoretical analysis of the worthiness of henry and elder problems as benchmarks of density-dependent groundwater flow models. Adv. Water Resour. 26 (1), 17-31.

Smith, A. J. \& TuRner, J. V. 2001 Density-dependent surface water-groundwater interaction and nutrient discharge in the Swan-Canning Estuary. Hydrol. Processes 15 (13), 2595-2616.

Taylor, T. P., Pennell, K. D., Abriola, L. M. \& Dane, J. H. 2001 Surfactant enhanced recovery of tetrachloroethylene from a porous medium containing low permeability lenses -1 . Experimental studies. J. Contaminant Hydrol. 48 (3-4), 325-350.

Voss, C. I. \& Provost, A. M. 2002 SUTRA, A model for saturated-unsaturated variable-density ground-water flow with solute or energy transport. T. Water-Resources Investigations Rep. 02-4231. US Geological Survey.

Weatherill, D., Simmons, C. T., Voss, C. I. \& Robinson, N. I. 2004 Testing density-dependent groundwater models: two-dimensional steady state unstable convection in infinite, finite and inclined porous layers. Adv. Water Resour. 27 (5), 548-563.

Wooding, R. A., TYler, S. W. \& White, I. 1997 Convection in groundwater below an evaporating salt lake. 1. Onset of instability. Water Resour. Res. 33, 1199-1217.

YANG, J. W. \& EDWARDS, R. N. 2000 Predicted groundwater circulation in fractured and unfractured anisotropic porous media driven by nuclear fuel waste heat generation. Can. J. Earth Sci. 37 (9), 1301-1308. 